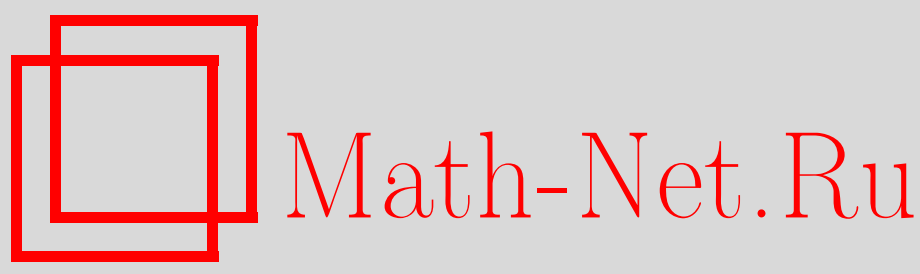

Ш. Т. Тетунашвили, О нуль-рядах по тригонометрической системе и по системам Уолша и Хаара, Матем. сб., 1996, том 187, номер 3, 103-142

DOI: https://doi.org/10.4213/sm119

Использование Общероссийского математического портала Math-Net.Ru подразумевает, что вы прочитали и согласны с пользовательским соглашением

http: //www. mathnet.ru/rus/agreement

Параметры загрузки:

IP : 3.85 .183 .62

26 апреля 2023 г., 07:46:21 
УДК 517.5

Ш. Т. Тетунашвили

\section{О нуль-рядах по тригонометрической системе и по системам Уолша и Хаара}

\footnotetext{
Решены некоторые задачи, связанные с нуль-рядами по тригонометрической системе и по системам Уолша и Хаара.

Библиография: 13 названий.
}

\section{Введение}

Будем рассматривать ряды по тригонометрической системе и по системам Уолша и Хаара.

Через $\left\{\varphi_{n}(t)\right\}$, где $t \in[a, b]$, обозначим одну из выше отмеченных систем.

В случае, когда $\left\{\varphi_{n}(t)\right\}$ - тригонометрическая система, то $[a, b]=[0,2 \pi]$, а когда $\left\{\varphi_{n}(t)\right\}$ - система Уолша или система Хаара, то $[a, b]=[0,1]$.

Рассмотрим ряд по системе $\left\{\varphi_{n}(t)\right\}$

$$
\sum_{n=1}^{\infty} a_{n} \varphi_{n}(t)
$$

Нуль-рядом по системе $\left\{\varphi_{n}(t)\right\}$ назьвается ряд (0.1), который почти всюду на $[a, b]$ сходится к нулю, хотя не все его коэффициенты равны нулю.

Первый пример тригонометрического нуль-ряда был построен Д.Е. Меньшовым [1]. Первый пример нуль-ряда по системе Уолша построен А. А. Шнейдером [2], а по системе Хаара - Г. Фабером [3].

Отметим, что нуль-ряд не может быть рядом Фурье, поэтому сумма квадратов коэффициентов нуль-ряда расходится.

I. Вопрос о том, существуют ли нуль-ряды (0.1), для которых

$$
\sum_{n=1}^{\infty} a_{n}^{2}
$$

расходится сколь угодно медленно, решается положительно как для тригонометрической системы (см. [4, с. 841]), так и для систем Уолша (см. [5]) и Хаара (см. [6]).

В данной работе отмеченные результаты усиливаются. Именно, мы докажем теоремы, из которых, в частности, следует, что для любой последовательности чисел $\left\{q_{n}\right\}$ с $0<q_{n} \leqslant 2$ при $n \geqslant 1$, сушествуют нуль-ряды как по тригонометрической системе, так и по системе Уолша, для которых ряд

$$
\sum_{n=1}^{\infty}\left|a_{n}\right|^{q_{n}}
$$

может расходиться сколь угодно медленно.

(C) Ш. Т. ТЕтунАшвили 1996 
II. Пусть $G(u)$ - функция, определенная на $[0,+\infty)$, а $\left\{\varepsilon_{n}\right\}$ - последовательность неотрицательных чисел. Через $S_{n}(t)$ обозначим частичные суммы ряда $(0.1)$.

Допустим, что для некоторой положительной постоянной $M$

$$
\int_{a}^{b}\left|S_{n}(t)\right|\left|G\left(\left|S_{n}(t)\right|\right)\right|^{\varepsilon_{n}} d t \leqslant M, \quad n=0,1, \ldots
$$

Как известно, если $G(u)=u$ и $\varepsilon_{n} \geqslant \varepsilon>0$ при $n \geqslant 0$, то из $(0.2)$ следует, что ряд (0.1) есть ряд Фурье.

Для тригонометрических рядов Литтлвудом была поставлена следующая проблема (см. $[7$, с. 5]).

Обязан ли тригонометрический ряд (0.1) быть рядом Фурье, если выполняется соотношение $(0.2)$ с $G(u)=1$ при $и \in[0,+\infty)$.

Эта проблема имеет отрицательное решение (см. [8]).

В связи с этим возник следующий, более общий вопрос, чем приведенная проблема Литтлвуда.

Существуют ли непрерьвная на $[0,+\infty)$ функция $G(u)$ и последовательность положительных чисел $\left\{\varepsilon_{n}\right\}$ c $\lim _{n \rightarrow \infty} \varepsilon_{n}=0$ такие, что при выполнении соотношения (0.2) следует, что ряд (0.1) есть ряд Фурье?

В данной работе докажем теоремы, из которых, в частности, следует отрицательный ответ на этот вопрос как для тригонометрических рядов, так и для рядов по системам Уолша и Хаара.

III. Пусть для любого $m \geqslant 0 \Omega_{m}=\left\{n: 2^{m}<n \leqslant 2^{m+1}\right\}$.

Рассмотрим ряд по системе Хаара

$$
\sum_{n=1}^{\infty} c_{n} \chi_{n}(t)
$$

Хорошо известен следуюший результат П. Л. Ульянова (см. [9]).

Пусть $d \geqslant 1$ - некоторое число. Если для последовательности $\left\{c_{n}\right\}$

$$
\max _{n \in \Omega_{m}}\left|c_{n}\right| \leqslant d \min _{n \in \Omega_{m-1}}\left|c_{n}\right| \quad(m=1,2, \ldots)
$$

и ряд (0.3) таков, что на некотором множестве $E \subset[0,1], \mu E>0$,

$$
\varlimsup_{m \rightarrow \infty}\left|\sum_{n=1}^{q_{m}} c_{n} \chi_{n}(t)\right|=M(t)<\infty
$$

при $t \in E$, где $q_{m}-$ некоторая возрастаюшая к $\infty$ последовательность целых чисел, то

$$
\sum_{n=1}^{\infty} c_{n}^{2}<\infty .
$$

П. Л. Ульянову принадлежит также (см. [10]) следуюшая 
ТЕОРема (о единственности для рядов по системе Хаара с монотонными коэффициентами). Если $c_{n} \geqslant c_{n+1} \geqslant 0$ при $n \geqslant 1$ и ряд (0.3) сходится к нулю на некотором множестве $E \subset[0,1], \mu E>0$, то существует натуральное число $n_{0}$ такое, что $c_{n}=0$ при $n \geqslant n_{0}$.

В той же работе П. Л. Ульяновым отмечено (см. [10, с. 73]), что в этой теореме условие монотонности коэффициентов можно ослабить. Например, теорема остается справедливой для рядов (0.3) с коэффициентами, удовлетворяющими более слабому условию, чем (0.4), и доказательство этого утверждения фактически содержится в работе [10].

Мы докажем теоремы о нуль-рядах по системе Хаара, из которых следует, что отмеченные результаты П. Л. Ульянова не могут быть усилены в определенном смысле. Одной из таких теорем является следуюшая

ТеоремА. Для любой последовательности $\left\{d_{m}\right\}, d_{m} \geqslant 1 u \lim _{m \rightarrow \infty} d_{m}=\infty$, существует нуль-ряд (0.3), коэффициенты которого удовлетворяют соотношениям: $c_{n}>0$ при $n \geqslant 1$ и

$$
\max _{n \in \Omega_{m}} c_{n} \leqslant d_{m} \min _{n \in \Omega_{m-1}} c_{n} \quad(m=1,2, \ldots) .
$$

Материал распределен следующим образом. В 11 даны обозначения, определения и сформулированы установленные нами теоремы. В $\S 2$ приведены известные результаты и установлены вспомогательные утверждения. В $\S 3$ доказываются теоремы, сформулированные в $\S 1$, и выводятся некоторые следствия этих теорем.

\section{§1. Обозначения, определения и формулировки результатов}

Пусть $\left\{r_{n}(t)\right\}_{n=0}^{\infty}-$ система Радемахера, где $t \in[0,1]$,

$$
r_{n}(t)=\operatorname{sign} \sin 2^{n+1} \pi t \quad(n=0,1,2, \ldots) ;
$$

$\left\{w_{n}(t)\right\}_{n=1}^{\infty}-$ система Уолша в нумерации Пэли, где $w_{1}(t) \equiv 1$, и если $n=1+$ $2^{m_{1}}+2^{m_{2}}+\cdots+2^{m_{s}}, 0 \leqslant m_{1}<\cdots<m_{s}$, то

$$
w_{n}(t)=\prod_{j=1}^{s} r_{m_{j}}(t) ;
$$

$\left\{\chi_{n}(t)\right\}_{n=1}^{\infty}$ - система Хаара, где $\chi_{1}(t) \equiv 1$, и если $n=2^{m}+j, 1 \leqslant j \leqslant 2^{m}$, то

$$
\chi_{n}(t)= \begin{cases}\sqrt{2^{m}} & \text { при } t \in\left(\frac{2 j-2}{2^{m+1}}, \frac{2 j-1}{2^{m+1}}\right), \\ -\sqrt{2^{m}} & \text { при } t \in\left(\frac{2 j-1}{2^{m+1}}, \frac{2 j}{2^{m+1}}\right), \\ 0 & \text { при остальных } t \in[0,1] .\end{cases}
$$

В дальнейшем будем пользоваться следуюшими обозначениями: для любого $m \geqslant 0$

$$
\Omega_{m}=\left\{n: 2^{m}<n \leqslant 2^{m+1}\right\}
$$


$\omega_{0}=\{1\}$, а если $m \geqslant 1$, то $\omega_{m}=\left\{n: p_{m-1}<n \leqslant p_{m}\right\}$, где $p_{m}=1+4+$ $\cdots+4^{m}=\frac{4^{m+1}-1}{3}$.

Пусть $\left\{d_{m}\right\}_{m=0}^{\infty}-$ любая последовательность положительных чисел с $d_{m} \uparrow \infty$, а $\Phi(u)$ - любая конечная на $[0,+\infty)$ функция с $\Phi(0)=0$.

Справедливы следуюшие утверждения.

Tеорема 1. Существует нуль-ряд

$$
1+\sum_{n=1}^{\infty} a_{n} \cos n \tau
$$

mакой, что $a_{n} \geqslant 0$ при $n \geqslant 1 u$

$$
\sum_{m=0}^{\infty} \frac{1}{d_{m}}\left|\Phi\left(\sum_{n \in \omega_{m}} a_{n}\right)\right| \leqslant 1 .
$$

Теорема 2. Существует нуль-ряд по системе Уолиа

$$
\sum_{n=1}^{\infty} b_{n} w_{n}(t)
$$

такой, что $b_{n} \geqslant 0$ при $n \geqslant 1 u$

$$
\sum_{m=0}^{\infty} \frac{1}{d_{m}}\left|\Phi\left(\sum_{n \in \Omega_{m}} b_{n}\right)\right| \leqslant 1 .
$$

Теорема 3. Существует нуль-ряд по системе Хаара

$$
\sum_{n=1}^{\infty} c_{n} \chi_{n}(t)
$$

mакой, что $c_{n} \geqslant 0$ nри $n \geqslant 1 u$

$$
\sum_{m=0}^{\infty} \frac{1}{d_{m}}\left|\Phi\left(\sqrt{2^{m}} \max _{n \in \Omega_{m}} c_{n}\right)\right| \leqslant 1
$$

Пусть, как и выше, $\left\{d_{m}\right\}$ - любая последовательность положительных чисел с $d_{m} \uparrow \infty$, а $\delta \in(0,1)$ - некоторое число. $\Psi(u)$ - любая конечная на $[0, \delta)$, неотрицательная функция со свойством: $\Psi(0)=0$ и для любых $\alpha \in[0, \delta), \beta \in[0, \delta)$

$$
\Psi(\alpha \beta) \leqslant \Psi(\alpha) \Psi(\beta) .
$$

Справедливы следующие утверждения. 
ТЕОрема 4. Существует нуль-ряд

$$
1+\sum_{n=1}^{\infty} a_{n} \cos n \tau
$$

maкой, что $0 \leqslant a_{n}<\delta$ nрu $n \geqslant 1 u$

$$
\sum_{m=0}^{\infty} \frac{1}{d_{m}} \sum_{n \in \omega_{m}} \Psi\left(a_{n}\right) \leqslant 1 .
$$

В частности, для любого $m \geqslant 1$

$$
\sum_{n=1}^{m} \Psi\left(a_{n}\right) \leqslant d_{m}
$$

ТЕОрема 5. Существует нуль-ряд по системе Уолиа

$$
\sum_{n=1}^{\infty} b_{n} w_{n}(t)
$$

такой, что $0 \leqslant b_{n}<\delta$ при $n \geqslant 1 u$

$$
\sum_{m=0}^{\infty} \frac{1}{d_{m}} \sum_{n \in \Omega_{m}} \Psi\left(b_{n}\right) \leqslant 1 .
$$

В частности, для любого $m>1$

$$
\sum_{n=2}^{m} \Psi\left(b_{n}\right) \leqslant d_{m} .
$$

Как уже отмечено (см. введение, пункт I), $\left\{\varphi_{n}(t)\right\}$, где $t \in[a, b]$, обозначает либо тригонометрическую систему, либо систему Уолша, либо систему Хаара. В случае, когда $\left\{\varphi_{n}(t)\right\}$ - тригонометрическая система, $[a, b]=[0,2 \pi]$, а в остальных случаях $[a, b]=[0,1] ; S_{m}(t)$ обозначает частичные суммы ряда

$$
\sum_{n=1}^{\infty} a_{n} \varphi_{n}(t)
$$

Справедливы следующие утверждения.

ТЕОРема 6. Пусть $\left\{G_{m}(u)\right\}_{m=1}^{\infty}-$ такая последовательность конечных на $[0,+\infty)$, неотрицательных и неубывающих функиий, что

$$
\sup _{u \geqslant 0} \varlimsup_{m \rightarrow \infty} G_{m}(u)<\infty .
$$

Тогда существует нуль-ряд (1.1) такой, что для любого $m \geqslant 1$

$$
\int_{a}^{b}\left|S_{m}(t)\right| G_{m}\left(\left|S_{m}(t)\right|\right) d t \leqslant M
$$

где $M$ - некоторое положительное число. 
ТЕОРЕМА 7. Для любой конечной, неотрищательной и неубьвающей на $[0,+\infty)$ функиии $G(u)$ и для любой последовательности чисел $\left\{\varepsilon_{m}\right\}, \varepsilon_{m}>0 u$ $\lim _{m \rightarrow \infty} \varepsilon_{m}=0$, существует нуль-ряд (1.1) такой, что при $m \geqslant 1$

$$
\int_{a}^{b}\left|S_{m}(t)\right|\left[G\left(\left|S_{m}(t)\right|\right)\right]^{\varepsilon_{m}} d t \leqslant M
$$

где $M$ - некоторое положсительное число.

Следуя обозначениям работы [9], через $A$ обозначим множество всех последовательностей $\left\{c_{n}\right\}$, для каждой из которых $c_{n} \geqslant c_{n+1} \geqslant 0$ при $n \geqslant 1$, а через $\bar{A}-$ множество всех последовательностей $\left\{c_{n}\right\}$, для каждой из которых найдется такое число $d \geqslant 1$, что выполняется соотношение (0.4).

Далее, пусть $\left\{l_{m}\right\}$ - некоторая последовательность целых чисел с $0 \leqslant l_{m} \leqslant m$ при $m \geqslant 0$.

Через $A\left(\left\{l_{m}\right\}\right)$ обозначим множество всех последовательностей $\left\{c_{n}\right\}$, для каждой из которых найдутся целочисленная последовательность $\left\{k_{m}\right\}$ и числа $d \geqslant 1$ и $q \geqslant 1$ такие, что если $m \geqslant q$, то

1) $0 \leqslant k_{m} \leqslant l_{m}$

и

2) $\max _{n \in \Omega_{m}}\left|c_{n}\right| \leqslant d \min _{n \in \Omega_{m-k_{m}}}\left|c_{n}\right|$.

Если для некоторого числа $m_{0}$ имеем $l_{m}=k$ при $m \geqslant m_{0}$, то $A\left(\left\{l_{m}\right\}\right)$ будем обозначать через $A(k)$.

Легко видеть, что

$$
A \subset \bar{A} \subset A(1) \subset A(2) \subset \cdots \subset A(k) \subset \cdots .
$$

Приведенная во введении (см. пункт III) теорема П. Л. Ульянова о единственности для рядов по системе Хаара

$$
\sum_{n=1}^{\infty} c_{n} \chi_{n}(t)
$$

с монотонными коэффициентами остается справедливой и для рядов (1.2), где

$$
\left\{c_{n}\right\} \in \bigcup_{k=1}^{\infty} A(k) .
$$

Доказательство этого утверждения, как мы уже заметили, фактически содержится в работе [10].

П. Л. Ульяновым был поставлен вопрос (см. [10, с. 73]) о максимальном расширении класса последовательностей $\left\{c_{n}\right\}$ так, чтобы эта теорема оставалась справедливой.

Ясно, что для любой последовательности $\left\{l_{m}\right\}$ с $\lim _{m \rightarrow \infty} l_{m}=\infty$

$$
\bigcup_{k=1}^{\infty} A(k) \subset A\left(\left\{l_{m}\right\}\right) .
$$

Следующими теоремами мы показываем, что приведенные выше результаты П. Л. Ульянова не могут быть усилены в определенном смысле. 
ТЕОРема 8. Для любой последовательности $\left\{l_{m}\right\}, \lim _{m \rightarrow \infty} l_{m}=\infty$, существует нуль-ряд (1.2) такой, что $c_{n}>0$ при $n \geqslant 1$ u $\left\{c_{n}\right\} \in A\left(\left\{l_{m}\right\}\right)$.

ТЕОРема 9. Для любой последовательности $\left\{d_{m}\right\}, d_{m} \geqslant 1 u \lim _{m \rightarrow \infty} d_{m}=\infty$, существует нуль-ряд (1.2) такой, что $c_{n}>0$ при $n \geqslant 1$ и

$$
\max _{n \in \Omega_{m}} c_{n} \leqslant d_{m} \min _{n \in \Omega_{m-1}} c_{n}
$$

где $m=1,2,3, \ldots$.

Отметим, что теоремы 8 и 9 были анонсированы нами в работе [13].

\section{§2. Известные результаты и вспомогательные утверждения}

Пусть $0<\lambda \leqslant 2$. Рассмотрим произведения Рисса

$$
\prod_{j=0}^{\infty}\left(1+\lambda \alpha_{j} \cos 4^{j} \tau\right)
$$

Говорят, что ряд

$$
1+\sum_{n=1}^{\infty} a_{n} \cos n \tau
$$

представляет произведение (2.1), если для любого $m \geqslant 0$

$$
\prod_{j=0}^{m}\left(1+\lambda \alpha_{j} \cos 4^{j} \tau\right)=1+\sum_{n=1}^{p_{m}} a_{n} \cos n \tau
$$

где

$$
p_{m}=1+4+\cdots+4^{m}=\frac{4^{m+1}-1}{3} .
$$

Рассмотрим произведение

$$
\prod_{j=0}^{\infty}\left(1+\alpha_{j} r_{j}(t)\right)
$$

Говорят, что ряд по системе Уолша

$$
\sum_{n=1}^{\infty} b_{n} w_{n}(t)
$$

представляет произведение (2.5), если для любого $m \geqslant 0$

$$
\prod_{j=0}^{m}\left(1+\alpha_{j} r_{j}(t)\right)=\sum_{n=1}^{2^{m+1}} b_{n} w_{n}(t)
$$


Будем говорить, что ряд по системе Хаара

$$
\sum_{n=1}^{\infty} c_{n} \chi_{n}(t)
$$

представляет произведение (2.5), если для любого $m \geqslant 0$

$$
\prod_{j=0}^{m}\left(1+\alpha_{j} r_{j}(t)\right)=\sum_{n=1}^{2^{m+1}} c_{n} \chi_{n}(t)
$$

Для частичных сумм рядов $(2.2),(2.6)$ и (2.8) введем обозначения

$$
\begin{aligned}
S_{m}(\tau) & =1+\sum_{n=1}^{m} a_{n} \cos n \tau, & & \tau \in[0,2 \pi] ; \\
W_{m}(t) & =\sum_{n=1}^{m} b_{n} w_{n}(t), & & t \in[0,1] ; \\
H_{m}(t) & =\sum_{n=1}^{m} c_{n} \chi_{n}(t), & & t \in[0,1] .
\end{aligned}
$$

Через $S$ будем обозначать множество всех последовательностей $\left\{\alpha_{j}\right\}$, для каждой из которых выполняются соотношения

1) $\sum_{j=0}^{\infty} \alpha_{j}^{2}=\infty$,

2) $\alpha_{j} \rightarrow \infty$ при $j \rightarrow \infty$,

3) $0 \leqslant \alpha_{j}<\frac{1}{2}$ при $j \geqslant 0$.

Для тригонометрических нуль-рядов Зигмундом (см. [11]; [12, с. 336]) была доказана теорема, которую мы сформулируем в удобном для нас виде.

Tеорема А (Зигмунд). Пусть $\left\{\alpha_{j}\right\} \in S u 0<\lambda \leqslant 2$.

Тогда ряд (2.2), представляющий произведение (2.1), почти всюду сходится к нулю.

Используя метод доказательства теоремы А, Шнейдер (см. [2]) построил нуль-ряд по системе Уолша. Именно, справедлива

Теорема В (Шнейдер). Если $\left\{\alpha_{j}\right\} \in S$, то ряд (2.6), представляющий произведение (2.5), почти всюду на $[0,1]$ сходится к нулю.

Пусть $\left\{\alpha_{j}\right\}_{j=0}^{\infty}-$ некоторая последовательность чисел и $B-$ подмножество из $\mathbb{Z}_{0}=\{0,1,2, \ldots\}$.

В дальнейшем, в случае $B=\varnothing$, будем считать, что

$$
\prod_{j \in B} \alpha_{j}=1 .
$$

Теперь докажем ряд лемм. Некоторые из них имеют самостоятельный интерес. 
Лемма 1. Пусть $\alpha_{j} \geqslant 0$ при $j \geqslant 0,0<\lambda \leqslant 2$, и тригонометрический ряд (2.2) представляет произведение (2.1). Тогда

а) все коэффициенты ряда (2.2) неотрицательны;

б) в случае $\lambda=2$ для коэффициентов ряда (2.2) выполняются следующие соотношения: при $m \geqslant 0$ для любого $n \in \omega_{m}$

$$
a_{n}=0 \quad \text { илu } \quad a_{n}=2 \alpha_{m} \prod_{j \in A_{n}^{(m-1)}} \alpha_{j}
$$

где $A_{n}^{(m-1)}$ - некоторое, возможно пустое, подмножсество из множества $\{0,1, \ldots, m-1\}$, зависящее лишь от $m$ и $n$. (Если $m=0$, то $n=1$. В этом случае считаем, что $A_{1}^{(-1)}=\varnothing$.)

ДоКАЗАТЕЛЬСТвО. В силу условия леммы при $m \geqslant 0$ имеем

$$
S_{p_{m}}(\tau)=1+\sum_{n=1}^{p_{m}} a_{n} \cos n \tau=\prod_{j=0}^{m}\left(1+\lambda \alpha_{j} \cos 4^{j} \tau\right),
$$

где $p_{m}=\frac{4^{m+1}-1}{3}$.

Поскольку $p_{m} \uparrow \infty$, то пункт а) будет доказан, если покажем, что для любого $m \geqslant 0$

$$
a_{n} \geqslant 0 \text { при } 1 \leqslant n \leqslant p_{m} .
$$

Соотношение (2.15) докажем методом математической индукции по $m$.

Пусть $m=0$. Тогда $p_{0}=1$, и поэтому из $(2.14)$ имеем

$$
1+a_{1} \cos \tau=1+\lambda \alpha_{0} \cos \tau
$$

Отсюда

$$
a_{1}=\lambda \alpha_{0}
$$

Поэтому

$$
a_{1} \geqslant 0,
$$

т.е. при $m=0$ соотношение (2.15) выполняется.

Допустим, что (2.15) выполняется для $m \leqslant k-1$ и покажем, что (2.15) справедливо и для $m=k$.

Из (2.14) следует, что

$$
\begin{aligned}
\sum_{n=p_{k-1}+1}^{p_{k}} a_{n} \cos n \tau & =S_{p_{k}}(\tau)-S_{p_{k-1}}(\tau) \\
& =\prod_{j=0}^{k}\left(1+\lambda \alpha_{j} \cos 4^{j} \tau\right)-\prod_{j=0}^{k-1}\left(1+\lambda \alpha_{j} \cos 4^{j} \tau\right) \\
& =\lambda \alpha_{k} \cos 4^{k} \tau \prod_{j=0}^{k-1}\left(1+\lambda \alpha_{j} \cos 4^{j} \tau\right)
\end{aligned}
$$


то есть

$$
\sum_{n \in \omega_{k}} a_{n} \cos n \tau=\lambda \alpha_{k} \cos 4^{k} \tau S_{p_{k-1}}(\tau)
$$

Поэтому

$$
\sum_{n \in \omega_{k}} a_{n} \cos n \tau=\lambda \alpha_{k} \cos 4^{k} \tau\left(1+\sum_{n=1}^{p_{k-1}} a_{n} \cos n \tau\right)
$$

Отсюда

$$
\sum_{n \in \omega_{k}} a_{n} \cos n \tau=\lambda \alpha_{k} \cos 4^{k} \tau+\frac{\lambda \alpha_{k}}{2} \sum_{n=1}^{p_{k-1}} a_{n}\left[\cos \left(4^{k}-n\right) \tau+\cos \left(4^{k}+n\right) \tau\right] .
$$

Поскольку $n \in \omega_{k}$, и $\omega_{k}=\left\{p_{k-1}+1, p_{k-1}+2, \ldots, p_{k}\right\}$, то возможны три случая

1) если $p_{k-1}<n<4^{k}-p_{k-1}$, то

$$
a_{n}=0
$$

2) если же $n=4^{k}$, то

$$
a_{4^{k}}=\lambda \alpha_{k},
$$

$3)$ если $n \neq 4^{k}$ и $4^{k}-p_{k-1} \leqslant n \leqslant 4^{k}+p_{k-1}=p_{k}$, то из (2.21) вытекает, что для этого числа $n$ сушествует число $q(n), 1 \leqslant q(n) \leqslant p_{k-1}$, такое, что

$$
a_{n}=\frac{\lambda \alpha_{k}}{2} a_{q(n)} .
$$

В силу предположения индукции $a_{q(n)} \geqslant 0$, поэтому из $(2.24)$ следует, что

$$
a_{n} \geqslant 0 \text {. }
$$

Из соотношений $(2.22),(2.23)$ и (2.25) вытекает справедливость пункта а).

Докажем пункт б). Пусть $m=0$; тогда $\omega_{0}=\{1\}$ и из соотношения (2.16), поскольку $\lambda=2$, имеем

$$
a_{1}=2 \alpha_{0}
$$

т.е. соотношение (2.13) при $m=0$ вьполняется.

Для $m \geqslant 1$ пункт б) докажем также методом математической индукции. Пусть $m=1$. Тогда

$$
\omega_{1}=\left\{n: p_{0}<n \leqslant p_{1}\right\}=\{2,3,4,5\} .
$$

Из (2.21) имеем

$$
\sum_{n \in \omega_{1}} a_{n} \cos n \tau=2 \alpha_{1} \cos 4 \tau+\alpha_{1} a_{1}(\cos 3 \tau+\cos 5 \tau) .
$$

Отсюда и из (2.26) имеем

$$
\sum_{n=2}^{5} a_{n} \cos n \tau=2 \alpha_{1} \alpha_{0} \cos 3 \tau+2 \alpha_{1} \cos 4 \tau+2 \alpha_{1} \alpha_{0} 5 \tau
$$


Отсюда при $m=1$ следует справедливость пункта б).

Допустим, что (2.13) выполняется при $m \leqslant k-1$ и покажем его справедливость для $m=k$.

Пусть $n \in \omega_{k}$. Тогда из соотношений (2.22) и (2.23) имеем:

1) если $p_{k-1}<n<4^{k}-p_{k-1}$, то

$$
a_{n}=0
$$

2) если $n=4^{k}$, то

$$
a_{4 k}=2 \alpha_{k}
$$

3) если $n \neq 4^{k}$ и $4^{k}-p_{k-1} \leqslant n \leqslant 4^{k}+p_{k-1}=p_{k}$, то из (2.24) имеем

$$
a_{n}=\alpha_{k} a_{q(n)}
$$

где $q(n) \in \omega_{i}$, для некоторого $i, 0 \leqslant i \leqslant k-1$.

В силу предположения индукции для $a_{q(n)}$ имеем:

$$
\begin{array}{ll}
\text { либо } & a_{q(n)}=0, \\
\text { либо } & a_{q(n)}=2 \alpha_{i} \prod_{j \in A_{q(n)}^{(i-1)}} \alpha_{j},
\end{array}
$$

где $A_{q(n)}^{(i-1)}$ - некоторое, возможно пустое, подмножество из $\{0,1, \ldots, i-1\}$.

Из (2.29)-(2.31) вытекает, что

$$
\begin{aligned}
& \text { либо } a_{n}=0 \text {, } \\
& \text { либо } a_{n}=2 \alpha_{k} \alpha_{i} \prod_{j \in A_{q(n)}^{(i-1)}} \alpha_{j} \text {. }
\end{aligned}
$$

Поскольку $i$ - некоторое целое число, изменяющееся от нуля до $k-1$, то очевидно, что соотношение (2.33) можно записать в виде

$$
a_{n}=2 \alpha_{k} \prod_{j \in A_{n}^{(k-1)}} \alpha_{j}
$$

где $A_{n}^{(k-1)}$ - некоторое подмножество из $\{0,1, \ldots, k-1\}$.

Из (2.27), (2.28), (2.32) и (2.34) следует справедливость пункта б). Лемма 1 доказана.

ЗАмечАниЕ 1 . Для любого $m \geqslant 1$ через $\omega_{m}^{1}$ будем обозначать множество всех чисел $n \in \omega_{m}$, для которых определено $A_{n}^{(m-1)}$. Из доказательства леммы 1 ясно, что $\omega_{m}^{1}$ не зависит от выбора последовательности $\left\{\alpha_{j}\right\}$, и если $n \in \omega_{m} \backslash \omega_{m}^{1}$, то $a_{n}=0$. 
Лемма 2. Пусть $\alpha_{j} \geqslant 0$ при $j \geqslant 0$ и ряд (2.6) представляет произведение (2.5). Тогда $b_{1}=1$, а для любого $n \in \Omega_{m}, m=0,1, \ldots$,

$$
b_{n}=\alpha_{m} \prod_{j \in B_{n}^{(m-1)}} \alpha_{j}
$$

где $B_{n}^{(m-1)}$ - некоторое, возможно пустое, подмножество из множества $\{0,1, \ldots, m-1\}$, зависящее лищь от $m$ и $n$. (Eсли $m=0$, то $n=2$. В этом случае считаем, что $B_{2}^{(-1)}=\varnothing$.) В частности, $b_{n} \geqslant 0$ для любого $n \geqslant 1$.

ДоКАЗАТЕЛЬСТВо. В силу условия леммы для любого $m \geqslant 0$

$$
\prod_{j=0}^{m}\left(1+\alpha_{j} r_{j}(t)\right)=\sum_{n=1}^{2^{m+1}} b_{n} w_{n}(t) .
$$

Отсюда при $m=0$ имеем

$$
1+\alpha_{0} r_{0}(t)=b_{1} w_{1}(t)+b_{2} w_{2}(t) .
$$

Поскольку $w_{1}(t) \equiv 1$ и $w_{2}(t)=r_{0}(t)$, то из $(2.37)$ имеем

$$
b_{1}=1 \text { и } b_{2}=\alpha_{0},
$$

т.е. при $m=0$ соотношение (2.35) выполняется.

Для $m \geqslant 1$ лемму докажем методом индукции. Из (2.36) имеем

$$
\sum_{n=2^{m}+1}^{2^{m+1}} b_{n} w_{n}(t)=\alpha_{m} r_{m}(t) \prod_{j=0}^{m-1}\left(1+\alpha_{j} r_{j}(t)\right) .
$$

Пусть $m=1$. Тогда из (2.39) имеем

$$
b_{3} w_{3}(t)+b_{4} w_{4}(t)=\alpha_{1} r_{1}(t)\left(1+\alpha_{0} r_{0}(t)\right)=\alpha_{1} w_{3}(t)+\alpha_{1} \alpha_{0} w_{4}(t) .
$$

Поэтому $b_{3}=\alpha_{1}$ и $b_{4}=\alpha_{1} \alpha_{0}$, т.е. при $m=1$ соотношение (2.35) выполняется.

Допустим, что лемма справедлива при $m \leqslant k-1$, и докажем ее для $m=k$.

Из (2.39) при $m=k$ получаем

$$
\sum_{n=2^{k}+1}^{2^{k+1}} b_{n} w_{n}(t)=\alpha_{k} r_{k}(t) \sum_{n=1}^{2^{k}} b_{n} w_{n}(t) .
$$

Если $n \in \Omega_{k}$, то

$$
n=2^{k}+q, \text { где } 1 \leqslant q \leqslant 2^{k} .
$$

В силу определения системы Уолша имеем

$$
w_{n}(t)=r_{k}(t) w_{q}(t)
$$


Из (2.40) и (2.41) ясно, что

$$
b_{n}=\alpha_{k} b_{q} .
$$

Поскольку $1 \leqslant q \leqslant 2^{k}$, то либо $q=1$, либо $q \in \Omega_{i}$ для некоторого $i, 0 \leqslant i \leqslant k-1$.

Поэтому, если $q=1$, то, так как $b_{1}=1$ (см. (2.38)), из (2.42) имеем

$$
b_{n}=\alpha_{k} .
$$

Если же $q \in \Omega_{i}$ для некоторого $i, 0 \leqslant i \leqslant k-1$, то в силу предположения индукции имеем

$$
b_{q}=\alpha_{i} \prod_{j \in B_{q}^{(i-1)}} \alpha_{j}
$$

где $B_{q}^{(i-1)}$ - некоторое, возможно пустое, подмножество из $\{0,1, \ldots, i-1\}$.

Из (2.42) и (2.44) следует, что

$$
b_{n}=\alpha_{k} \alpha_{i} \prod_{j \in B_{q}^{(i-1)}} \alpha_{j} .
$$

Поскольку $i$ - некоторое число от нуля до $k-1$, то очевидно, что соотношение (2.45) можно записать в виде

$$
b_{n}=\alpha_{k} \prod_{j \in B_{n}^{(k-1)}} \alpha_{j},
$$

где $B_{n}^{(k-1)}$ - некоторое подмножество из $\{0,1, \ldots, k-1\}$.

Из $(2.38),(2.43)$ и (2.46) следует справедливость соотношения (2.35).

Лемма 2 доказана.

Для $n=2^{m}+k$, где $1 \leqslant k \leqslant 2^{m}$, обозначим

$$
\operatorname{supp} \chi_{n}=\left(\frac{k-1}{2^{m}}, \frac{k}{2^{m}}\right) \text {. }
$$

Справедлива следуюшая

Лемма 3. Пусть $0 \leqslant \alpha_{j}<1$ при $j \geqslant 0$ и ряд (2.8) представляет произведение (2.5). Тогда $c_{1}=1, c_{2}=\alpha_{0}$ и для любого $n \in \Omega_{m} \quad(m=1,2, \ldots)$

$$
c_{n}=\frac{\alpha_{m}}{\sqrt{2^{m}}} \prod_{j=0}^{m-1}\left(1+\alpha_{j} r_{j}(t)\right), \quad \text { əde } \quad t \in \operatorname{supp} \chi_{n} .
$$

В частности,

$$
\begin{aligned}
& \max _{n \in \Omega_{m}} c_{n}=c_{2^{m}+1}=\frac{\alpha_{m}}{\sqrt{2^{m}}} \prod_{j=0}^{m-1}\left(1+\alpha_{j}\right), \\
& \min _{n \in \Omega_{m}} c_{n}=c_{2^{m+1}}=\frac{\alpha_{m}}{\sqrt{2^{m}}} \prod_{j=0}^{m-1}\left(1-\alpha_{j}\right)
\end{aligned}
$$

$u c_{n} \geqslant 0$ для любого $n \geqslant 1$. 
ДОКАЗАТЕЛЬСТВо. В силу условия леммы для любого $m \geqslant 0$ имеем

$$
\prod_{j=0}^{m}\left(1+\alpha_{j} r_{j}(t)\right)=\sum_{n=1}^{2^{m+1}} c_{n} \chi_{n}(t) .
$$

При $m=0$ из (2.50) получаем

$$
1+\alpha_{0} r_{0}(t)=c_{1} \chi_{1}(t)+c_{2} \chi_{2}(t) .
$$

Поскольку $\chi_{1}(t) \equiv 1$ и $\chi_{2}(t)=r_{0}(t)$, то из $(2.51)$ следует

$$
c_{1}=1 \text { и } c_{2}=\alpha_{0} .
$$

Далее, для любого $m \geqslant 1$ из (2.50) следует, что

$$
\sum_{n=2^{m}+1}^{2^{m+1}} c_{n} \chi_{n}(t)=\alpha_{m} r_{m}(t) \prod_{j=0}^{m-1}\left(1+\alpha_{j} r_{j}(t)\right) \text {. }
$$

Из определения систем Хаара и Радемахера имеем

$$
r_{m}(t)=\frac{1}{\sqrt{2^{m}}} \sum_{n \in \Omega_{m}} \chi_{n}(t), \quad m=0,1,2, \ldots
$$

Из (2.53) и (2.54) получаем

$$
\begin{aligned}
\sum_{n=2^{m}+1}^{2^{m+1}} c_{n} \chi_{n}(t)= & \left(\frac{\alpha_{m}}{\sqrt{2^{m}}} \prod_{j=0}^{m-1}\left(1+\alpha_{j} r_{j}(t)\right)\right) \chi_{2^{m}+1}(t) \\
& +\left(\frac{\alpha_{m}}{\sqrt{2^{m}}} \prod_{j=0}^{m-1}\left(1+\alpha_{j} r_{j}(t)\right)\right) \chi_{2^{m}+2}(t)+\cdots \\
& \cdots+\left(\frac{\alpha_{m}}{\sqrt{2^{m}}} \prod_{j=0}^{m-1}\left(1+\alpha_{j} r_{j}(t)\right)\right) \chi_{2^{m+1}}(t) .
\end{aligned}
$$

Ясно, что если $t \in \operatorname{supp} \chi_{2^{m}+k}$, где $1 \leqslant k \leqslant 2^{m}$, то для любого фиксированного $j, 0 \leqslant j \leqslant m-1$,

$$
r_{j}(t)=\text { const },
$$

поэтому при $t \in \operatorname{supp} \chi_{2^{m}+k}, 1 \leqslant k \leqslant 2^{m}$, имеем

$$
\prod_{j=0}^{m-1}\left(1+\alpha_{j} r_{j}(t)\right)=\text { const } .
$$

Из (2.55) и (2.56) следует, что справедливо (2.47). 
При $t \in \operatorname{supp} \chi_{2^{m}+1}$ имеем

$$
r_{j}(t)=1, \quad 0 \leqslant j \leqslant m-1
$$

при $t \in \operatorname{supp} \chi_{2^{m+1}}$ имеем

$$
r_{j}(t)=-1, \quad 0 \leqslant j \leqslant m-1
$$

Поэтому, так как $0 \leqslant \alpha_{j}<1$, из $(2.55)$ и (2.57) имеем

$$
\max _{n \in \Omega_{m}} c_{n}=c_{2^{m}+1}=\frac{\alpha_{m}}{\sqrt{2^{m}}} \prod_{j=0}^{m-1}\left(1+\alpha_{j}\right),
$$

а из (2.55) и (2.58) следует, что

$$
\min _{n \in \Omega_{m}} c_{n}=c_{2^{m+1}}=\frac{\alpha_{m}}{\sqrt{2^{m}}} \prod_{j=0}^{m-1}\left(1-\alpha_{j}\right) .
$$

Лемма 3 доказана.

В дальнейшем, при $m=0$ будем считать, что

$$
\prod_{j=0}^{m-1} \alpha_{j}=1
$$

ЛЕмма 4. а) Если тригонометрический ряд (2.2) представляет произведение (2.1), то для любого $m \geqslant 0$

$$
\sum_{n \in \omega_{m}} a_{n}=\lambda \alpha_{m} \prod_{j=0}^{m-1}\left(1+\lambda \alpha_{j}\right)
$$

б) Если ряд (2.6) представляет произведение (2.5), то для любого $m \geqslant 0$

$$
\sum_{n \in \Omega_{m}} b_{n}=\alpha_{m} \prod_{j=0}^{m-1}\left(1+\alpha_{j}\right) .
$$

в) Если ряд (2.8) представляет произведение (2.5), то для любого $m \geqslant 0$

$$
\sqrt{2^{m}} c_{2^{m}+1}=\alpha_{m} \prod_{j=0}^{m-1}\left(1+\alpha_{j}\right)
$$

ДокАЗАТЕЛЬство. В равенстве (2.18), взяв $\tau=0$, получаем (2.59), а в соотношениях $(2.39)$ и $(2.53)$, взяв точку $t \in\left(0, \frac{1}{2^{m+1}}\right)$, получаем $(2.60)$ и $(2.61)$ соответственно.

Лемма 4 доказана. 
Лемма 5. Пусть $A_{n}^{(m-1)}$ и $B_{n}^{(m-1)}-$ множествва из лемм 1 и 2. Тогда для любой последовательности $\left\{\alpha_{j}\right\}_{j=0}^{\infty}$ справедливы следующие соотношения

$$
\begin{array}{lll}
\sum_{n \in \omega_{m}^{1}} 2 \alpha_{m} & \prod_{j \in A_{n}^{(m-1)}} \alpha_{j}=2 \alpha_{m} \prod_{j=0}^{m-1}\left(1+2 \alpha_{j}\right), & m=0,1, \ldots, \\
\text { б) } \sum_{n \in \Omega_{m}} \alpha_{m} \prod_{j \in B_{n}^{(m-1)}} \alpha_{j}=\alpha_{m} \prod_{j=0}^{m-1}\left(1+\alpha_{j}\right), & m=0,1, \ldots
\end{array}
$$

ДоКАЗАТЕЛЬСтво. Из равенства (2.59) для $\lambda=2$, из (2.13) и из замечания 1 следует (2.62), а соотношение (2.63) следует из (2.35) и (2.60).

Лемма 5 доказана.

Лемма 6. Пусть $\left\{\alpha_{j}\right\}_{j=0}^{\infty} \in S$ и ряд (2.8) представляет произведение (2.5). Тогда ряд (2.8) есть нуль-ряд по системе Хаара.

Кроме того, для частичных сумм ряда (2.8) имеем $H_{p}(t)>0$ для любого $t \in[0,1]$ u $p \geqslant 1$.

ДокАЗАТЕЛЬство. Пусть ряд (2.6) по системе Уолша представляет произведение (2.5), т.е.

$$
\sum_{n=0}^{2^{m+1}} b_{n} w_{n}(t)=\prod_{j=0}^{m}\left(1+\alpha_{j} r_{j}(t)\right), \quad m=0,1, \ldots
$$

Поскольку $\left\{\alpha_{j}\right\} \in S$, то в силу теоремы В, в частности, имеем

$$
\lim _{m \rightarrow \infty} \sum_{n=0}^{2^{m+1}} b_{n} w_{n}(t)=0, \text { п.в. } t \in[0,1] .
$$

В силу условия леммы, для любого $m \geqslant 0$

$$
H_{2^{m+1}}(t)=\sum_{n=1}^{2^{m+1}} c_{n} \chi_{n}(t)=\prod_{j=0}^{m}\left(1+\alpha_{j} r_{j}(t)\right) .
$$

Из (2.64), (2.66) и (2.65) следует, что

$$
\lim _{m \rightarrow \infty} H_{2^{m+1}}(t)=0 \text { для п.в. } t \in[0,1] .
$$

Так как для любого $t \in[0,1]$ и $1 \leqslant k \leqslant 2^{m}$ имеем

$$
H_{2^{m}+k}(t)=\left\{H_{2^{m}}(t) \text { либо } H_{2^{m+1}}(t)\right\},
$$

то из (2.68) и (2.67) следует

$$
\lim _{p \rightarrow \infty} H_{p}(t)=0 \text { для п.в. } t \in[0,1],
$$


т.е. $\sum_{n=1}^{\infty} c_{n} \chi_{n}(t)$ есть нуль-ряд.

Далее, так как $0 \leqslant \alpha_{j}<1 / 2$, то

$$
\prod_{j=0}^{m}\left(1+\alpha_{j} r_{j}(t)\right)>0 \text { для } t \in[0,1] .
$$

Из (2.69) и (2.66) следует, что

$$
H_{2^{m+1}}(t)>0 \text { при } t \in[0,1] \text { и } m \geqslant 0 .
$$

В силу (2.70) и (2.68) имеем

$$
H_{p}(t)>0 \text { при } t \in[0,1] \text { и } p \geqslant 2,
$$

а поскольку $c_{1}=1$ (см. лемму 3 ), то (2.71) выполняется и для $p=1$.

Лемма 6 доказана.

ЛЕмма 7. Для любой последовательности положсительных чисел $\left\{d_{m}\right\}_{m=0}^{\infty}$, $d_{m} \uparrow \infty$, любой конечной на $[0,+\infty)$ функиии $\Phi, \Phi(0)=0$, существует последовательность $\left\{\alpha_{j}\right\}_{j=0}^{\infty} \in S$ такая, ито

$$
\sum_{m=0}^{\infty} \frac{1}{d_{m}}\left|\Phi\left(\alpha_{m} \prod_{j=0}^{m-1}\left(1+\alpha_{j}\right)\right)\right| \leqslant 1 .
$$

ДокАЗАТЕЛьство. Определим последовательность натуральных чисел $\left\{m_{k}\right\}_{k=0}^{\infty}$ следующим образом.

Пусть $m_{0}$ - некоторое число, для которого

$$
d_{m_{0}}>2\left|\Phi\left(\frac{1}{\sqrt{9}}\right)\right|
$$

а для любого $k \geqslant 1$ число $m_{k}$ определим так

$$
\begin{gathered}
m_{k}>m_{k-1}, \\
d_{m_{k}}>2^{k+1}\left|\Phi\left(\frac{1}{\sqrt{k+9}} \prod_{j=0}^{k-1}\left(1+\frac{1}{\sqrt{j+9}}\right)\right)\right|
\end{gathered}
$$

(поскольку $d_{m} \uparrow \infty$, то ясно, что такие $m_{k}$ существуют).

Теперь определим последовательность $\left\{\alpha_{j}\right\}_{j=0}^{\infty}$ :

$$
\alpha_{j}= \begin{cases}0, & \text { если } j \neq m_{k} \text { при } k \geqslant 0, \\ \frac{1}{\sqrt{k+9}}, & \text { если } j=m_{k} .\end{cases}
$$

Из (2.74) ясно, что

1) $\sum_{j=0}^{\infty} \alpha_{j}^{2}=\infty$

2) $\lim _{j \rightarrow \infty} \alpha_{j}=0$,

3) $0 \leqslant \alpha_{j}<1 / 2$ при $j \geqslant 0$, т.е.

$$
\left\{\alpha_{j}\right\}_{j=0}^{\infty} \in S
$$


Из (2.74) следует, что при $m \geqslant 0$

$$
\alpha_{m} \prod_{j=0}^{m-1}\left(1+\alpha_{j}\right)=\left\{\begin{array}{l}
0, \text { если } m \neq m_{k} \text { при } k \geqslant 0, \\
\frac{1}{\sqrt{k+9}} \prod_{j=0}^{k-1}\left(1+\frac{1}{\sqrt{j+9}}\right), \quad \text { если } m=m_{k} .
\end{array}\right.
$$

Поскольку $\Phi(0)=0$, то из $(2.76)$ следует, что

$$
\begin{aligned}
& \Phi\left(\alpha_{m} \prod_{j=0}^{m-1}\left(1+\alpha_{j}\right)\right) \\
& \quad=\left\{\begin{array}{l}
0, \text { если } m \neq m_{k} \text { при } k \geqslant 0, \\
\Phi\left(\frac{1}{\sqrt{k+9}} \prod_{j=0}^{k-1}\left(1+\frac{1}{\sqrt{j+9}}\right)\right), \text { если } m=m_{k} .
\end{array}\right.
\end{aligned}
$$

Из (2.77) и (2.73) ясно, что

$$
\begin{aligned}
& \sum_{m=0}^{\infty} \frac{1}{d_{m}}\left|\Phi\left(\alpha_{m} \prod_{j=0}^{m-1}\left(1+\alpha_{j}\right)\right)\right| \\
& \quad=\sum_{k=0}^{\infty} \frac{1}{d_{m_{k}}}\left|\Phi\left(\frac{1}{\sqrt{k+9}} \prod_{j=0}^{k-1}\left(1+\frac{1}{\sqrt{j+9}}\right)\right)\right| \leqslant \sum_{k=0}^{\infty} \frac{1}{2^{k+1}}=1 .
\end{aligned}
$$

\section{Лемма 7 доказана.}

Через $S_{0}$ обозначим множество всех последовательностей $\left\{\alpha_{j}\right\}$ из $S$, для каждой из которых $0<\alpha_{j}<1 / 2$ при $j \geqslant 0$.

Лемма 8. Для любой последовательности $\left\{\tau_{m}\right\}, \tau_{m} \geqslant 0 u \lim _{m \rightarrow \infty} \tau_{m}=\infty$, существует последовательность $\left\{\alpha_{j}\right\} \in S_{0}$ такая, что при $m \geqslant 1$

$$
\begin{gathered}
\alpha_{m} \leqslant \sqrt[4]{2} \alpha_{m-1}, \\
\prod_{j=0}^{m-1} \frac{1+\alpha_{j}}{1-\alpha_{j}}<2^{\left(1+\tau_{m}\right) / 4} .
\end{gathered}
$$

ДоКАЗАТЕЛЬСтво. Сначала отметим, что если $0<\alpha_{j}<1 / 2$ при $j \geqslant 0$, то

$$
\prod_{j=0}^{m-1} \frac{1+\alpha_{j}}{1-\alpha_{j}}<2^{8 \sum_{j=0}^{m-1} \alpha_{j}}, \quad m=1,2, \ldots
$$

Действительно, так как $0<\alpha_{j}<1 / 2$, то при $m \geqslant 1$

$$
\prod_{j=0}^{m-1} \frac{1+\alpha_{j}}{1-\alpha_{j}}=\prod_{j=0}^{m-1}\left(1+\frac{2 \alpha_{j}}{1-\alpha_{j}}\right)<\prod_{j=0}^{m-1}\left(1+4 \alpha_{j}\right) .
$$


Поскольку при $t>0 \ln (1+t)<t$, то

$$
\prod_{j=0}^{m-1}\left(1+4 \alpha_{j}\right)=e^{\sum_{j=0}^{m-1} \ln \left(1+4 \alpha_{j}\right)}<e^{4 \sum_{j=0}^{m-1} \alpha_{j}}<2^{8 \sum_{j=0}^{m-1} \alpha_{j}}
$$

Из (2.81) и (2.82) следует (2.80).

Определим последовательность неотрицательных целых чисел $\left\{m_{k}\right\}_{k=0}^{\infty}$ : пусть $m_{0}=0$, а для любого $k \geqslant 1$ число $m_{k}$ такое, что $m_{k}>m_{k-1}$ и

$$
\min _{m>m_{k}} \tau_{m} \geqslant k
$$

Для любого $k \geqslant 1$ положим

$$
\Delta_{k}=\left\{m: m_{k-1}<m \leqslant m_{k}\right\} .
$$

Ясно, что $m_{k} \uparrow \infty$, поэтому

$$
\bigcup_{k=1}^{\infty} \Delta_{k}=\{1,2,3, \ldots\}
$$

Далее, пусть для любого $m \in \Delta_{k}$

$$
\tau_{m}^{\prime}=k-1 \quad(k=1,2, \ldots) .
$$

Из соотношений (2.83)-(2.86) очевидно, что для любого $m \geqslant 1$

$$
\tau_{m}^{\prime} \leqslant \tau_{m}
$$

Теперь построим последовательность $\left\{\alpha_{m}^{\prime}\right\}_{m=0}^{\infty}$ такую, что

$$
\left\{\alpha_{m}^{\prime}\right\}_{m=0}^{\infty} \in S_{0}
$$

и для любого $m \geqslant 1$

$$
\alpha_{m}^{\prime} \leqslant \sqrt[4]{2} \alpha_{m-1}^{\prime}
$$

и

$$
\sum_{j=0}^{m-1} \alpha_{j}^{\prime} \leqslant 1+\tau_{m}
$$

Если $0 \leqslant m \leqslant m_{1}$, то

$$
\alpha_{m}^{\prime}=\frac{1}{2 m_{1}}
$$

Для любого $k \geqslant 2$

$$
\alpha_{m_{k}}^{\prime}=\frac{1-q}{2 m_{1} \sqrt{k}}, \quad \text { где } q=\frac{1}{\sqrt[4]{2}} .
$$

Далее, если $m \in \Delta_{k}$ при $k \geqslant 2$, то

$$
\alpha_{m}^{\prime}=\alpha_{m_{k}}^{\prime} q^{m_{k}-m} .
$$


Таким образом, определена последовательность $\left\{\alpha_{m}^{\prime}\right\}_{m=0}^{\infty}$. Покажем, что для этой последовательности выполняются соотношения (2.88), (2.89) и (2.90).

Для любого $m \in \Delta_{k}$ из (2.93) и (2.92) следует, что

$$
0<\alpha_{m}^{\prime} \leqslant \alpha_{m_{k}}^{\prime}=\frac{1-q}{2 m_{1} \sqrt{k}}
$$

Отсюда ясно, что для любого $m \geqslant 0$

$$
0<\alpha_{m}^{\prime}<\frac{1}{2}
$$

и

$$
\lim _{m \rightarrow \infty} \alpha_{m}^{\prime}=0
$$

Далее,

$$
\sum_{m=0}^{\infty}\left(\alpha_{m}^{\prime}\right)^{2} \geqslant \sum_{k=2}^{\infty}\left(\alpha_{m_{k}}^{\prime}\right)^{2}=\sum_{k=2}^{\infty} \frac{(1-q)^{2}}{4 m_{1}^{2} k}=\frac{(1-q)^{2}}{4 m_{1}^{2}} \sum_{k=2}^{\infty} \frac{1}{k}=\infty
$$

Соотношения (2.94)-(2.96) означают, что выполняется (2.88).

Покажем, что выполняется (2.89). Если $m-1 \in \Delta_{k}$ и $m \in \Delta_{k}$, то из (2.93) следует

$$
\alpha_{m-1}^{\prime}=\alpha_{m_{k}}^{\prime} q^{m_{k}-(m-1)}
$$

и

$$
\alpha_{m}^{\prime}=\alpha_{m_{k}}^{\prime} q^{m_{k}-m}
$$

Отсюда имеем

$$
\alpha_{m}^{\prime}=\sqrt[4]{2} \alpha_{m-1}^{\prime}
$$

Если $m-1 \in \Delta_{k}$ и $m \in \Delta_{k+1}$, то $m-1=m_{k}$, т.е.

$$
\alpha_{m-1}^{\prime}=\alpha_{m_{k}}^{\prime}
$$

Поскольку $m \in \Delta_{k+1}$, то

$$
\alpha_{m}^{\prime}=\alpha_{m_{k+1}}^{\prime} q^{m_{k+1}-m} \leqslant \alpha_{m_{k+1}}^{\prime} \leqslant \alpha_{m_{k}}^{\prime}=\alpha_{m-1}^{\prime} .
$$

Объединяя (2.97) и (2.98) получаем, что при $m \geqslant 1$

$$
\alpha_{m}^{\prime} \leqslant \sqrt[4]{2} \alpha_{m-1}^{\prime}
$$

т.е. (2.89) выполняется.

Покажем теперь, что для любого $m \geqslant 1$

$$
\sum_{j=0}^{m-1} \alpha_{j}^{\prime} \leqslant 1+\tau_{m}^{\prime}
$$


Пусть $m \in \Delta_{k}$. Тогда в силу (2.86) $\tau_{m}^{\prime}=k-1$, и поэтому соотношение (2.99) равносильно неравенству

$$
\sum_{j=0}^{m-1} \alpha_{j}^{\prime} \leqslant k
$$

Покажем, что выполняется (2.100).

Пусть $k=1$. Тогда, поскольку $m \leqslant m_{1}$, из (2.91) имеем

$$
\sum_{j=0}^{m-1} \alpha_{j}^{\prime}<\sum_{j=0}^{m_{1}} \alpha_{j}^{\prime}=\sum_{j=0}^{m_{1}} \frac{1}{2 m_{1}}=\frac{m_{1}+1}{2 m_{1}} \leqslant 1
$$

т.е. при $k=1$ соотношение (2.100) выполняется.

Если же $k \geqslant 2$, то так как $m \in \Delta_{k}$ и $\alpha_{j}^{\prime}>0$ при $j \geqslant 0$, то имеем

$$
\sum_{j=0}^{m-1} \alpha_{j}^{\prime}<\sum_{j=0}^{m_{k}} \alpha_{j}^{\prime}=\sum_{j=0}^{m_{1}} \alpha_{j}^{\prime}+\sum_{l=2}^{k} \sum_{j \in \Delta_{l}} \alpha_{j}^{\prime} .
$$

Для любого $l, 2 \leqslant l \leqslant k$, из (2.93) имеем

$$
\begin{aligned}
\sum_{j \in \Delta_{l}} \alpha_{j}^{\prime} & =\alpha_{m_{l}}^{\prime} \sum_{j \in \Delta_{l}} q^{m_{l}-j} \leqslant \alpha_{m_{l}}^{\prime}\left(1+q+q^{2}+\cdots\right) \\
& =\alpha_{m_{l}}^{\prime} \frac{1}{1-q}=\frac{1-q}{2 m_{1} \sqrt{l}} \frac{1}{1-q}=\frac{1}{2 m_{1} \sqrt{l}}<1 .
\end{aligned}
$$

Из (2.102), (2.101) и (2.103) следует, что если $m \in \Delta_{k}$, то

$$
\sum_{j=0}^{m-1} \alpha_{j}^{\prime} \leqslant k
$$

Это неравенство означает, что выполняется (2.99).

Из (2.99) и (2.87) получаем, что

$$
\sum_{j=0}^{m-1} \alpha_{j}^{\prime} \leqslant 1+\tau_{m}
$$

Справедливость соотношения (2.90) показана.

Пусть для любого $m \geqslant 0$

$$
\alpha_{m}=\frac{\alpha_{m}^{\prime}}{32} .
$$

Покажем, что последовательность $\left\{\alpha_{m}\right\}_{m=0}^{\infty}$ удовлетворяет всем требованиям леммы 8.

Действительно, так как $\left\{\alpha_{m}^{\prime}\right\}_{m=0}^{\infty} \in S_{0}$, то из (2.104) следует, что $\left\{\alpha_{m}\right\}_{m=0}^{\infty} \in$ $S_{0}$. Из (2.89) и (2.104) следует, что при $m \geqslant 1$

$$
\alpha_{m} \leqslant \sqrt[4]{2} \alpha_{m-1}
$$


Далее, из (2.90) и (2.104) при $m \geqslant 1$ имеем

$$
32 \sum_{j=0}^{m-1} \alpha_{j} \leqslant 1+\tau_{m} .
$$

Отсюда

$$
8 \sum_{j=0}^{m-1} \alpha_{j} \leqslant \frac{1+\tau_{m}}{4} .
$$

Из (2.80) и (2.105) получаем, что

$$
\prod_{j=0}^{m-1} \frac{1+\alpha_{j}}{1-\alpha_{j}}<2^{\left(1+\tau_{m}\right) / 4} .
$$

Лемма 8 доказана.

Лемма 9. Для любой последовательности $\left\{d_{m}\right\}_{m=1}^{\infty}, d_{m} \geqslant 1 u \lim _{m \rightarrow \infty} d_{m}=\infty$, существует последовательность $\left\{\alpha_{j}\right\} \in S$ такая, ито при $m \geqslant 1$

a) $\prod_{j=0}^{m}\left(1+2 \alpha_{j}\right) \leqslant d_{m}$

б) $\alpha_{m}^{2} \prod_{j=0}^{m-1}\left(1+2 \alpha_{j}^{2}\right) \leqslant 1$.

ДокАЗАТЕЛЬСТво. Пусть $m_{1}$ - такое натуральное число, что

$$
\text { если } m>m_{1}, \text { то } d_{m}>e^{2} \text {. }
$$

Далее, для любого $k \geqslant 2$ число $m_{k}$ определим так:

$$
m_{k}>m_{k-1} \text { и если } m>m_{k} \text {, то } d_{m}>e^{k+1} \text {. }
$$

Последовательность $\left\{\alpha_{j}\right\}$ построим следуюшим образом:

$$
\text { если } 0 \leqslant j \leqslant m_{1}, \text { то } \alpha_{j}=0 .
$$

Далее, если $j>m_{1}$, то

$$
\alpha_{j}= \begin{cases}0, & \text { если } j \neq m_{k}, k=2,3, \ldots, \\ \frac{1}{3 \sqrt{k}}, & \text { если } j=m_{k} .\end{cases}
$$

Очевидно, что $\left\{\alpha_{j}\right\}_{j=0}^{\infty} \in S$. Если $0 \leqslant m \leqslant m_{1}$, то

$$
\prod_{j=0}^{m}\left(1+2 \alpha_{j}\right)=1
$$

и пункт а) выполняется. 
Пусть $m>m_{1}$. Тогда $m_{k-1}<m \leqslant m_{k}$ для некоторого $k \geqslant 2$. Поэтому

$$
\prod_{j=0}^{m}\left(1+2 \alpha_{j}\right) \leqslant \prod_{j=0}^{m_{k}}\left(1+2 \alpha_{j}\right)=\prod_{j=2}^{k}\left(1+\frac{2}{3 \sqrt{j}}\right) .
$$

Поскольку $1+t \leqslant e^{t}$ при $t \geqslant 0$, то

$$
\prod_{j=2}^{k}\left(1+\frac{2}{3 \sqrt{j}}\right) \leqslant e^{\sum_{j=2}^{k} \frac{2}{3 \sqrt{3}}}<e^{k} .
$$

Далее, поскольку $m>m_{k-1}$, то из (2.106) имеем

$$
e^{k}<d_{m}
$$

Из (2.107), (2.108) и (2.109) получаем, что при $m \geqslant 1$

$$
\prod_{j=0}^{m}\left(1+2 \alpha_{j}\right)<d_{m}
$$

и пункт а) доказан.

Если $1 \leqslant m \leqslant m_{1}$, то из определения $\left\{\alpha_{j}\right\}$ следует, что

$$
\alpha_{m}^{2} \prod_{j=0}^{m-1}\left(1+2 \alpha_{j}^{2}\right)=0
$$

и пункт б) выполняется.

Если же $m_{k-1}<m \leqslant m_{k}$ для $k \geqslant 2$, то

$$
\begin{aligned}
\alpha_{m}^{2} \prod_{j=0}^{m-1}\left(1+2 \alpha_{j}^{2}\right) & \leqslant \alpha_{m_{k}}^{2} \prod_{j=0}^{m_{k}}\left(1+2 \alpha_{j}^{2}\right)=\frac{1}{9 k} \prod_{j=2}^{k}\left(1+\frac{2}{9 j}\right) \\
& \leqslant \frac{1}{9 k} e^{\frac{2}{9} \sum_{j=2}^{k} \frac{1}{j}} \leqslant \frac{1}{9 k} e^{\frac{2}{9} \ln k} \leqslant 1 .
\end{aligned}
$$

Лемма 9 доказана.

Если $P(t)$ - полином по системе $\{\cos n t\}_{n=0}^{\infty}$, то

$$
\|P\|_{L_{1}}=\int_{0}^{2 \pi}|P(t)| d t \quad \text { и }\|P\|_{c}=\sup _{0 \leqslant t \leqslant 2 \pi}|P(t)|,
$$

а если $P(t)$ - полином по системе Уолша или по системе Хаара, то

$$
\|P\|_{L_{1}}=\int_{0}^{1}|P(t)| d t \quad \text { и }\|P\|_{c}=\sup _{0 \leqslant t \leqslant 1}|P(t)| .
$$

Пусть $\left\{d_{m}\right\}$ - любая последовательность чисел с

$$
d_{m} \geqslant 1 \text { и } \lim _{m \rightarrow \infty} d_{m}=\infty .
$$

Справедливы следующие утверждения. 
Лемма 10. Существует нуль-ряд

$$
1+\sum_{n=1}^{\infty} a_{n} \cos n \tau
$$

с частичными суммами $S_{m}(\tau)$ такой, что при $m \geqslant 1$

a) $\left\|S_{m}\right\|_{c} \leqslant d_{m}$,

б) $\left\|S_{m}\right\|_{L_{1}} \leqslant 16$.

Лемма 11. Существует нуль-ряд по системе Уолша

$$
\sum_{n=1}^{\infty} b_{n} w_{n}(t)
$$

с частичными суммами $W_{m}(t)$ такой, что при $m \geqslant 1$

a) $\left\|W_{m}\right\|_{c} \leqslant d_{m}$

б) $\left\|W_{m}\right\|_{L_{1}} \leqslant 2$.

Лемма 12. Существует нуль-ряд по системе Хаара

$$
\sum_{n=1}^{\infty} c_{n} \chi_{n}(t)
$$

с частичныцми суммами $H_{m}(t)$ такой, что при $m \geqslant 1$

a) $\left\|H_{m}\right\|_{c} \leqslant d_{m}$,

б) $\left\|H_{m}\right\|_{L_{1}}=1$.

Доказательства леммы 10 и леммы 11 фактически не отличаются. Поэтому мы докажем лемму 10 и лемму 12.

ДОКАЗАТЕЛЬСТво ЛЕММЫ 10 . Пусть $\omega_{0}=\{1\}$ и для $m \geqslant 1 \omega_{m}=\left\{n: p_{m-1}<\right.$ $\left.n \leqslant p_{m}\right\}$, где $p_{m}=\frac{4^{m+1}-1}{3}$. Для любого $m \geqslant 1$ обозначим

$$
q_{m}=\min _{k \in \omega_{m}} d_{k} .
$$

Очевидно, что $q_{m} \geqslant 1$ при $m \geqslant 1$ и

$$
\lim _{m \rightarrow \infty} q_{m}=\infty .
$$

Поэтому в силу леммы 9 сушествует последовательность $\left\{\alpha_{j}\right\} \in S$ такая, что при $m \geqslant 1$

$$
\prod_{j=0}^{m}\left(1+2 \alpha_{j}\right) \leqslant q_{m}
$$

и

$$
\alpha_{m}^{2} \prod_{j=0}^{m-1}\left(1+2 \alpha_{j}^{2}\right) \leqslant 1 .
$$


Пусть для этой последовательности $\left\{\alpha_{j}\right\}$ ряд (2.2) представляет произведение (2.1) с $\lambda=2$, т.е. для любого $m \geqslant 0$

$$
\prod_{j=0}^{m}\left(1+2 \alpha_{j} \cos 4^{j} \tau\right)=1+\sum_{n=1}^{p_{m}} a_{n} \cos n \tau
$$

Тогда в силу теоремы А ряд (2.2) есть нуль-ряд.

Пусть $k \geqslant 1$ - любое натуральное число. Тогда $k \in \omega_{m}$ для некоторого $m \geqslant 0$. В силу леммы $1 a_{n} \geqslant 0$ при $n \geqslant 1$, и поэтому

$$
\left\|S_{k}\right\|_{c}=S_{k}(0)=1+\sum_{n=1}^{k} a_{n} \leqslant 1+\sum_{n=1}^{p_{m}} a_{n} .
$$

В $(2.113)$, взяв $\tau=0$, получаем

$$
\prod_{j=0}^{m}\left(1+2 \alpha_{j}\right)=1+\sum_{n=1}^{p_{m}} a_{n}
$$

Из (2.114) и (2.115) получаем, что

$$
\left\|S_{k}\right\|_{c} \leqslant \prod_{j=0}^{m}\left(1+2 \alpha_{j}\right)
$$

Из (2.116) и (2.111) следует, что

$$
\left\|S_{k}\right\|_{c} \leqslant q_{m}
$$

Из (2.110) очевидно, что для любого $k \in \omega_{m}$

$$
q_{m} \leqslant d_{k}
$$

Из (2.117) и (2.118) следует, что при $k \geqslant 1\left\|S_{k}\right\|_{c} \leqslant d_{k}$.

Пункт а) доказан.

Далее, пусть $k \in \omega_{m}$ для некоторого $m \geqslant 1$. Тогда

$$
\begin{aligned}
\left\|S_{k}\right\|_{L_{1}} & =\left\|S_{p_{m-1}}+\left(S_{k}-S_{p_{m-1}}\right)\right\|_{L_{1}} \\
& \leqslant\left\|S_{p_{m-1}}\right\|_{L_{1}}+\left\|S_{k}-S_{p_{m-1}}\right\|_{L_{1}} \\
& \leqslant\left\|S_{p_{m-1}}\right\|_{L_{1}}+\sqrt{2 \pi}\left\|S_{k}-S_{p_{m-1}}\right\|_{L_{2}} .
\end{aligned}
$$

Из соотношения (2.113) очевидно, что при $m \geqslant 1 S_{p_{m-1}}(\tau) \geqslant 0$ для любого $\tau \in$ $[0,2 \pi]$. Поэтому для $m \geqslant 1$

$$
\left\|S_{p_{m-1}}\right\|_{L_{1}}=\int_{0}^{2 \pi} S_{p_{m-1}}(\tau) d \tau=2 \pi .
$$


Ясно, что

$$
\left\|S_{k}-S_{p_{m-1}}\right\|_{L_{2}} \leqslant\left(\pi \sum_{n \in \omega_{m}} a_{n}^{2}\right)^{1 / 2} .
$$

В силу леммы 1 и замечания 1 имеем

$$
\sum_{n \in \omega_{m}} a_{n}^{2}=\sum_{n \in \omega_{m}^{1}} a_{n}^{2}=\sum_{n \in \omega_{m}^{1}} 4 \alpha_{m}^{2} \prod_{j \in A_{n}^{(m-1)}} \alpha_{j}^{2} .
$$

Используя лемму 5 , получаем, что при $m \geqslant 1$

$$
\sum_{n \in \omega_{m}^{1}} 4 \alpha_{m}^{2} \prod_{j \in A_{n}^{(m-1)}} \alpha_{j}^{2}=4 \alpha_{m}^{2} \prod_{j=0}^{m-1}\left(1+2 \alpha_{j}^{2}\right) .
$$

Из (2.121), (2.122) и (2.123) следует, что при $m \geqslant 1$

$$
\left\|S_{k}-S_{p_{m-1}}\right\|_{L_{2}} \leqslant\left(\pi 4 \alpha_{m}^{2} \prod_{j=0}^{m-1}\left(1+2 \alpha_{j}^{2}\right)\right)^{1 / 2} .
$$

Из (2.112) и (2.124) следует, что

$$
\left\|S_{k}-S_{p_{m-1}}\right\|_{L_{2}} \leqslant 2 \sqrt{\pi}
$$

Из (2.119), (2.120) и (2.125) получаем, что

$$
\left\|S_{k}\right\|_{L_{1}} \leqslant 2 \pi+2 \sqrt{2} \pi<16
$$

Лемма 10 доказана.

ДоКАЗАТЕЛЬСТво ЛЕМмЫ 12. Для Любого $m \geqslant 0$ обозначим

$$
q_{m}=\min _{2^{m}<k \leqslant 2^{m+1}} d_{k}
$$

Очевидно, что $q_{m} \geqslant 1$ при $m \geqslant 1$ и

$$
\lim _{m \rightarrow \infty} q_{m}=\infty
$$

Поэтому в силу леммы 9 существует последовательность $\left\{\alpha_{j}\right\} \in S$ такая, что при $m \geqslant 1$

$$
\prod_{j=0}^{m}\left(1+\alpha_{j}\right) \leqslant q_{m}
$$

Пусть для этой последовательности $\left\{\alpha_{j}\right\}$, ряд (2.8) представляет произведение (2.5), т.е. при $m \geqslant 0$

$$
\prod_{j=0}^{m}\left(1+\alpha_{j} r_{j}(t)\right)=\sum_{n=1}^{2^{m+1}} c_{n} \chi_{n}(t)
$$


Тогда в силу леммы 6 ряд (2.8) есть нуль-ряд.

Пусть $k \geqslant 2$ - любое натуральное число. Тогда $2^{m}<k \leqslant 2^{m+1}$ для некоторого $m \geqslant 0$. Поэтому для любого $t \in[0,1]$

$$
H_{k}(t)=\left\{H_{2^{m}}(t) \text { либо } H_{2^{m+1}}(t)\right\} .
$$

Поскольку $\alpha_{j} \geqslant 0$ при $j \geqslant 0$, то из (2.128) следует, что

$$
\left\|H_{2^{m+1}}\right\|_{c}=\prod_{j=0}^{m}\left(1+\alpha_{j}\right) .
$$

Отсюда ясно, что

$$
\left\|H_{2^{m}}\right\|_{c} \leqslant\left\|H_{2^{m+1}}\right\|_{c}
$$

Из (2.129), (2.130) и (2.131) следует, что

$$
\left\|H_{k}\right\|_{c} \leqslant\left\|H_{2^{m+1}}\right\|_{c}=\prod_{j=0}^{m}\left(1+\alpha_{j}\right) .
$$

Из (2.127) и (2.132) получаем

$$
\left\|H_{k}\right\|_{c} \leqslant q_{m}
$$

В силу (2.126) очевидно, что для любого $k$ при $2^{m}<k \leqslant 2^{m+1}$

$$
q_{m} \leqslant d_{k}
$$

Из (2.133) и (2.134) следует, что при $k \geqslant 2$

$$
\left\|H_{k}\right\|_{c} \leqslant d_{k}
$$

В случае $k=1$ имеем: $H_{1}(t)=c_{1}=1$ (см. лемму 3) т.е. (2.135) выполняется и при $k=1$.

Пункт а) доказан.

Далее, в силу леммы 6 для любого $k \geqslant 1$

$$
H_{k}(t)>0 \text { при } t \in[0,1]
$$

и поскольку $c_{1}=1$, то

$$
\left\|H_{k}\right\|_{L_{1}}=\int_{0}^{1} H_{k}(t) d t=1
$$

Лемма 12 доказана. 
ЛЕмма 13. Пусть $\left\{G_{m}(u)\right\}$ такая последовательность конечных на $[0,+\infty)$ функиий, что

$$
\sup _{u \geqslant 0} \varlimsup_{m \rightarrow \infty} G_{m}(u)<\infty .
$$

Тогда существуют число $M>0$ и последовательность чисел $\left\{d_{m}\right\}_{m=1}^{\infty}$ такие, что для любого $m \geqslant 1$

$$
d_{m} \geqslant 1, \quad \lim _{m \rightarrow \infty} d_{m}=\infty \quad u \quad G_{m}\left(d_{m}\right) \leqslant M .
$$

ДокАЗАТЕЛЬСТво. В силу условия

$$
\sup _{u \geqslant 0} \varlimsup_{m \rightarrow \infty} G_{m}(u)=N<\infty .
$$

Ясно, что для любого $u \geqslant 0$

$$
\varlimsup_{m \rightarrow \infty} G_{m}(u) \leqslant N .
$$

Отсюда следует, что сушествует число $m_{1}$ такое, что если $m \geqslant m_{1}$, то $G_{m}(1) \leqslant$ $N+1$. Для любого $k \geqslant 2$ число $m_{k}$ определим так: $m_{k}>m_{k-1}$ и если $m>m_{k}$, то

$$
G_{m}(k) \leqslant N+1 .
$$

Ясно, что $m_{k} \uparrow \infty$ при $k \rightarrow \infty$. Последовательность $\left\{d_{m}\right\}$ определим следующим образом

$$
d_{m}=\left\{\begin{array}{l}
1, \text { если } 1 \leqslant m \leqslant m_{1}, \\
k, \text { если } m_{k} \leqslant m<m_{k+1}, k=2,3, \ldots .
\end{array}\right.
$$

Поскольку $m_{k} \uparrow \infty$, то числа $d_{m}$ определены для любого $m \geqslant 1$ и очевидно, что $d_{m} \geqslant 1$ и $d_{m} \uparrow \infty$.

Покажем, что для любого $m \geqslant m_{1}$

$$
G_{m}\left(d_{m}\right) \leqslant N+1
$$

Действительно, если $m \geqslant m_{1}$, то существует число $k \geqslant 1$ такое, что $m_{k} \leqslant m<$ $m_{k+1}$.

В этом случае, по определению (см. (2.137) и (2.136)),

$$
d_{m}=k \text { и } \quad G_{m}(k) \leqslant N+1 .
$$

Отсюда следует справедливость соотношения (2.138). Пусть

$$
M=\max \left\{\max _{1 \leqslant m \leqslant m_{1}} G_{m}(1) ; N+1\right\} .
$$

Тогда, поскольку $d_{m}=1$ при $1 \leqslant m \leqslant m_{1}$, и при $m \geqslant m_{1}$ выполняется (2.138), получаем, что для любого $m \geqslant 1$

$$
G_{m}\left(d_{m}\right) \leqslant M .
$$

Лемма 13 доказана. 


\section{§3. Доказательства теорем и их следствий}

ДОКАЗАТЕЛЬСТВА ТЕОРЕМ 1,2 и 3. В силу лемМы 7 для последовательности положительных чисел $\left\{d_{m}\right\}$ с $d_{m} \uparrow \infty$ и любой конечной на $[0,+\infty)$ функции $\Phi(u)$ с $\Phi(0)=0$ сушествует последовательность $\left\{\alpha_{j}\right\}_{j=0}^{\infty} \in S$ такая, что

$$
\sum_{m=0}^{\infty} \frac{1}{d_{m}}\left|\Phi\left(\alpha_{m} \prod_{j=0}^{m-1}\left(1+\alpha_{j}\right)\right)\right| \leqslant 1 .
$$

Пусть для этой последовательности $\left\{\alpha_{j}\right\}_{j=0}^{\infty}$ ряд (2.2) представляет произведение (2.1) с $\lambda=1$, а ряды (2.6) и (2.8) представляют произведение (2.5). Тогда в силу теоремы А, теоремы В и леммы 6 для рядов $(2.2),(2.6)$ и (2.8) имеем

$$
\begin{aligned}
1+\sum_{n=1}^{\infty} a_{n} \cos n \tau & =0 \text { для п.в. } \tau \in[0,2 \pi], \\
\sum_{n=1}^{\infty} b_{n} w_{n}(t) & =0 \text { для п.в. } t \in[0,1], \\
\sum_{n=1}^{\infty} c_{n} \chi_{n}(t) & =0 \text { для п.в. } t \in[0,1] .
\end{aligned}
$$

Кроме того, в силу лемм 1,2 и 3 для любого $n \geqslant 1$

$$
a_{n} \geqslant 0, \quad b_{n} \geqslant 0, \quad c_{n} \geqslant 0 .
$$

Из леммы 4 (см. (2.59), (2.60)) и леммы 3 (см. (2.48)) для любого $m \geqslant 0$ получаем

$$
\sum_{n \in \omega_{m}} a_{n}=\sum_{n \in \Omega_{m}} b_{n}=\sqrt{2^{m}} \max _{n \in \Omega_{m}} c_{n}=\alpha_{m} \prod_{j=0}^{m-1}\left(1+\alpha_{j}\right)
$$

Отсюда в силу (3.1) ясно, что для любого $N \geqslant 0$

$$
\begin{aligned}
\sum_{m=0}^{N} \frac{1}{d_{m}}\left|\Phi\left(\sum_{n \in \omega_{m}} a_{n}\right)\right| & =\sum_{m=0}^{N} \frac{1}{d_{m}}\left|\Phi\left(\sum_{n \in \Omega_{m}} b_{n}\right)\right| \\
& =\sum_{m=0}^{N} \frac{1}{d_{m}}\left|\Phi\left(\sqrt{2^{m}} \max _{n \in \Omega_{m}} c_{n}\right)\right| \\
& =\sum_{m=0}^{N} \frac{1}{d_{m}}\left|\Phi\left(\alpha_{m} \prod_{j=0}^{m-1}\left(1+\alpha_{j}\right)\right)\right| \leqslant 1 .
\end{aligned}
$$

Из соотношений (3.2)-(3.6) следует справедливость теорем 1,2 и 3. 
ДОКАЗАТЕЛЬСТВА ТЕОРЕМ 4 и 5. Определим последовательность натуральных чисел $\left\{m_{k}\right\}_{k=0}^{\infty}$ следуюшим образом.

Пусть $m_{0}$ - такое натуральное число, что

$$
d_{m_{0}}>2\left[\Psi\left(\frac{\delta}{\sqrt{9}}\right)+\Psi\left(\frac{2 \delta}{\sqrt{9}}\right)\right]
$$

а для любого $k \geqslant 1$, число $m_{k}$ определим так: $m_{k}>m_{k-1}$ и

$$
d_{m_{k}}>2^{k+1}\left[\Psi\left(\frac{\delta}{\sqrt{k+9}}\right)+\Psi\left(\frac{2 \delta}{\sqrt{k+9}}\right)\right] \prod_{j=0}^{k-1}\left(1+2 \Psi\left(\frac{\delta}{\sqrt{j+9}}\right)\right) \text {. }
$$

Отсюда, поскольку $\Psi(u) \geqslant 0$ при $u \in[0, \delta)$, то для $k \geqslant 0$ имеем

$$
\begin{aligned}
& d_{m_{k}}>2^{k+1} \Psi\left(\frac{2 \delta}{\sqrt{k+9}}\right) \prod_{j=0}^{k-1}\left(1+2 \Psi\left(\frac{\delta}{\sqrt{j+9}}\right)\right) \\
& d_{m_{k}}>2^{k+1} \Psi\left(\frac{\delta}{\sqrt{k+9}}\right) \prod_{j=0}^{k-1}\left(1+\Psi\left(\frac{\delta}{\sqrt{j+9}}\right)\right) .
\end{aligned}
$$

Теперь определим последовательность $\left\{\alpha_{j}\right\}_{j=0}^{\infty}$ :

$$
\alpha_{j}= \begin{cases}0, & \text { если } j \neq m_{k} \text { при } k \geqslant 0, \\ \frac{\delta}{\sqrt{k+9}}, & \text { если } j=m_{k} .\end{cases}
$$

Легко видеть, что $\left\{\alpha_{j}\right\} \in S$ и для любого $j \geqslant 0$

$$
0 \leqslant \alpha_{j}<\frac{\delta}{3}
$$

Пусть для этой последовательности $\left\{\alpha_{j}\right\}$ ряд

$$
1+\sum_{n=1}^{\infty} a_{n} \cos n \tau
$$

представляет произведение

$$
\prod_{j=0}^{\infty}\left(1+2 \alpha_{j} \cos 4^{j} \tau\right)
$$

а ряд

$$
\sum_{n=1}^{\infty} b_{n} w_{n}(t)
$$

представляет произведение

$$
\prod_{j=0}^{\infty}\left(1+\alpha_{j} r_{j}(t)\right)
$$


Тогда в силу теорем А и В следует, что ряды (2.2) и (2.6) являются нуль-рядами.

В силу леммы 1 для любого $n \in \omega_{m}$ при $m \geqslant 0$

$$
a_{n}=0 \text { или } a_{n}=2 \alpha_{m} \prod_{j \in A_{n}^{(m-1)}} \alpha_{j},
$$

а в силу леммы 2 для любого $n \in \Omega_{m}$ при $m \geqslant 0$

$$
b_{n}=\alpha_{m} \prod_{j \in B_{n}^{(m-1)}} \alpha_{j}
$$

Из (3.8) и (3.9) следует, что при $n \geqslant 1$

$$
0 \leqslant a_{n}<\delta
$$

а из $(3.8)$ и $\left(3.9^{\prime}\right)$ получаем, что при $n \geqslant 2$

$$
0 \leqslant b_{n}<\delta \text {. }
$$

Используя свойство функций $\Psi$, из (3.9) получим, что для любого $n \in \omega_{m}$, где $m \geqslant 0$,

$$
\Psi\left(a_{n}\right)=0 \text { или } \Psi\left(a_{n}\right) \leqslant \Psi\left(2 \alpha_{m}\right) \prod_{j \in A_{n}^{(m-1)}} \Psi\left(\alpha_{j}\right),
$$

а из $\left(3.9^{\prime}\right)$ получаем, что для любого $n \in \Omega_{m}$, где $m \geqslant 0$,

$$
\Psi\left(b_{n}\right) \leqslant \Psi\left(\alpha_{m}\right) \prod_{j \in B_{n}^{(m-1)}} \Psi\left(\alpha_{j}\right) .
$$

Из (3.10), из пункта а) леммы 5 и замечания 1 получаем, что при $m \geqslant 0$

$$
\sum_{n \in \omega_{m}} \Psi\left(a_{n}\right) \leqslant \sum_{n \in \omega_{m}^{1}} \Psi\left(2 \alpha_{m}\right) \prod_{j \in A_{n}^{(m-1)}} \Psi\left(\alpha_{j}\right)=\Psi\left(2 \alpha_{m}\right) \prod_{j=0}^{m-1}\left(1+2 \Psi\left(\alpha_{j}\right)\right),
$$

а из $\left(3.10^{\prime}\right)$ и из пункта б) леммы 5 следует, что при $m \geqslant 0$

$$
\sum_{n \in \Omega_{m}} \Psi\left(b_{n}\right) \leqslant \sum_{n \in \Omega_{m}} \Psi\left(\alpha_{m}\right) \prod_{j \in B_{n}^{(m-1)}} \Psi\left(\alpha_{j}\right)=\Psi\left(\alpha_{j}\right) \prod_{j=0}^{m-1}\left(1+\Psi\left(\alpha_{j}\right)\right) .
$$

Из определения последовательности $\left\{\alpha_{j}\right\}$, поскольку $\Psi(0)=0$, следует, что при $m \geqslant 0$

$$
\begin{aligned}
& \Psi\left(2 \alpha_{m}\right) \prod_{j=0}^{m-1}\left(1+2 \Psi\left(\alpha_{j}\right)\right) \\
& =\left\{\begin{array}{l}
0, \text { если } m \neq m_{k} \text { при } k \geqslant 0, \\
\Psi\left(\frac{2 \delta}{\sqrt{k+9}}\right) \prod_{j=0}^{k-1}\left(1+2 \Psi\left(\frac{\delta}{\sqrt{j+9}}\right)\right), \text { если } m=m_{k},
\end{array}\right. \\
& \Psi\left(\alpha_{m}\right) \prod_{j=0}^{m-1}\left(1+\Psi\left(\alpha_{j}\right)\right) \\
& =\left\{\begin{array}{l}
0, \text { если } m \neq m_{k} \text { при } k \geqslant 0, \\
\Psi\left(\frac{\delta}{\sqrt{k+9}}\right) \prod_{j=0}^{k-1}\left(1+\Psi\left(\frac{\delta}{\sqrt{j+9}}\right)\right), \text { если } m=m_{k} .
\end{array}\right.
\end{aligned}
$$


Из (3.12) и (3.7) получаем, что

$$
\begin{aligned}
& \sum_{m=0}^{\infty} \frac{1}{d_{m}} \Psi\left(2 \alpha_{m}\right) \prod_{j=0}^{m-1}\left(1+2 \Psi\left(\alpha_{j}\right)\right) \\
& \quad=\sum_{k=0}^{\infty} \frac{1}{d_{m_{k}}} \Psi\left(\frac{2 \delta}{\sqrt{k+9}}\right) \prod_{j=0}^{k-1}\left(1+2 \Psi\left(\frac{\delta}{\sqrt{j+9}}\right)\right) \\
& \quad \leqslant \sum_{k=0}^{\infty} \frac{1}{2^{k+1}}=1 .
\end{aligned}
$$

Из $\left(3.12^{\prime}\right)$ и $\left(3.7^{\prime}\right)$ следует

$$
\begin{aligned}
& \sum_{m=0}^{\infty} \frac{1}{d_{m}} \Psi\left(\alpha_{m}\right) \prod_{j=0}^{m-1}\left(1+\Psi\left(\alpha_{j}\right)\right) \\
& \quad=\sum_{k=0}^{\infty} \frac{1}{d_{m_{k}}} \Psi\left(\frac{\delta}{\sqrt{k+9}}\right) \prod_{j=0}^{k-1}\left(1+\Psi\left(\frac{\delta}{\sqrt{j+9}}\right)\right) \\
& \quad \leqslant \sum_{k=0}^{\infty} \frac{1}{2^{k+1}}=1 .
\end{aligned}
$$

Из (3.11) и (3.13) следует, что

$$
\sum_{m=0}^{\infty} \frac{1}{d_{m}} \sum_{n \in \omega_{m}} \Psi\left(a_{n}\right) \leqslant 1,
$$

а из $\left(3.11^{\prime}\right)$ и $\left(3.13^{\prime}\right)$ имеем

$$
\sum_{m=0}^{\infty} \frac{1}{d_{m}} \sum_{n \in \Omega_{m}} \Psi\left(b_{n}\right) \leqslant 1 .
$$

Поскольку $d_{m} \uparrow \infty$, то из $(3.14)$ и $\left(3.14^{\prime}\right)$ следует, что для любого $N \geqslant 1$

$$
\begin{aligned}
& \frac{1}{d_{N}} \sum_{m=0}^{N} \sum_{n \in \omega_{m}} \Psi\left(a_{n}\right) \leqslant \sum_{m=0}^{N} \frac{1}{d_{m}} \sum_{n \in \omega_{m}} \Psi\left(a_{n}\right) \leqslant 1, \\
& \frac{1}{d_{N}} \sum_{m=0}^{N} \sum_{n \in \Omega_{m}} \Psi\left(b_{n}\right) \leqslant \sum_{m=0}^{N} \frac{1}{d_{m}} \sum_{n \in \Omega_{m}} \Psi\left(b_{n}\right) \leqslant 1 .
\end{aligned}
$$

Из $(3.15)$ и $\left(3.15^{\prime}\right)$ очевидно, что для любого $N \geqslant 1$

$$
\begin{aligned}
& \sum_{m=1}^{N} \Psi\left(a_{n}\right) \leqslant d_{N}, \\
& \sum_{m=2}^{N} \Psi\left(b_{n}\right) \leqslant d_{N}, \quad N \geqslant 2 .
\end{aligned}
$$

Теоремы 4 и 5 доказаны. 
ДОКАЗАТЕЛЬСТВО ТЕОРЕМЫ 6 . В силУ леммЫ 13 сушествуют число $M>0$ и последовательность чисел $\left\{d_{m}\right\}_{m=1}^{\infty}$ такие, что при $m \geqslant 1$

$$
d_{m} \geqslant 1, \quad \lim _{m \rightarrow \infty} d_{m}=\infty
$$

и

$$
G_{m}\left(d_{m}\right) \leqslant M .
$$

Для этой последовательности $\left\{d_{m}\right\}$ в силу леммы 10 сушествует нуль-ряд

$$
1+\sum_{n=1}^{\infty} a_{n} \cos n \tau
$$

с частичньми суммами $S_{m}(\tau)$ такой, что при $m \geqslant 1$

$$
\begin{aligned}
& \sup _{0 \leqslant \tau \leqslant 2 \pi}\left|S_{m}(\tau)\right| \leqslant d_{m}, \\
& \int_{0}^{2 \pi}\left|S_{m}(\tau)\right| d \tau \leqslant 16 .
\end{aligned}
$$

Отсюда, поскольку $G_{m}(u)$ неубывающая функция для любого $m \geqslant 1$, получаем

$$
\begin{aligned}
\int_{0}^{2 \pi}\left|S_{m}(\tau)\right| G_{m}\left(\left|S_{m}(\tau)\right|\right) d \tau & \leqslant \int_{0}^{2 \pi}\left|S_{m}(\tau)\right| G_{m}\left(d_{m}\right) d \tau \\
& \leqslant M \int_{0}^{2 \pi}\left|S_{m}(\tau)\right| d \tau \leqslant 16 M
\end{aligned}
$$

т.е. теорема справедлива для тригонометрических нуль-рядов.

Точно так же, с помошью леммы 11 вместо леммы 10, теорема доказывается для нуль-рядов по системе Уолша, а применяя лемму 12 вместо леммы 10, доказываем справедливость теоремы и для нуль-рядов по системе Хаара. Теорема 6 доказана.

ДОКАЗАТЕЛЬСТВО ТЕОРЕМЫ 7. ПУСть дЛя ЛюбОГО $m \geqslant 1$

$$
G_{m}(u)=[G(u)]^{\varepsilon_{m}} .
$$

Тогда из условия теоремы очевидно, что для любого $m \geqslant 1$

1) $0 \leqslant G_{m}(u)<\infty$ при $u \in[0,+\infty)$,

2) если $u_{1}<u_{2}$, то $G_{m}\left(u_{1}\right) \leqslant G_{m}\left(u_{2}\right)$.

Далее, ясно, что для любого фиксированного $u \in[0,+\infty)$

$$
\lim _{m \rightarrow \infty} G_{m}(u)=\lim _{m \rightarrow \infty}[G(u)]^{\varepsilon_{m}}=\left\{\begin{array}{l}
0, \text { если } G(u)=0, \\
1, \text { если } G(u)>0,
\end{array}\right.
$$

и поэтому

$$
\sup _{u \geqslant 0} \lim _{m \rightarrow \infty} G_{m}(u) \leqslant 1,
$$

т.е. последовательность $\left\{G_{m}(u)\right\}$ удовлетворяет требованиям теоремы 6 . Отсюда и следует справедливость теоремы 7. 
ДОКАЗАТЕЛЬСТВО ТЕОРЕМЫ 8 . Поскольку $l_{m} \geqslant 0$ и $\lim _{m \rightarrow \infty} l_{m}=\infty$, то в силу леммы 8 сушествует последовательность $\left\{\alpha_{j}\right\} \in S_{0}$ (т.е. $\left\{\alpha_{j}\right\} \in S$ и $\alpha_{j}>0$ при $j \geqslant 0)$ такая, что при $m \geqslant 1$

$$
\begin{gathered}
\alpha_{m} \leqslant \sqrt[4]{2} \alpha_{m-1}, \\
\prod_{j=0}^{m-1} \frac{1+\alpha_{j}}{1-\alpha_{j}} \leqslant 2^{\left(1+l_{m}\right) / 4} .
\end{gathered}
$$

Пусть для этой последовательности $\left\{\alpha_{j}\right\}_{j=0}^{\infty}$ ряд

$$
\sum_{n=1}^{\infty} c_{n} \chi_{n}(t)
$$

представляет произведение (2.5). Тогда, в силу леммы 6, ряд (2.8) есть нуль-ряд, а из леммы 3 (см. (2.48) и (2.49)) следует, что $c_{n}>0$ при $n \geqslant 1$ (поскольку $0<\alpha_{j}<\frac{1}{2}$ при $j \geqslant 0)$ и

$$
\begin{aligned}
\max _{n \in \Omega_{m}} c_{n} & =\frac{\alpha_{m}}{\sqrt{2^{m}}} \prod_{j=0}^{m-1}\left(1+\alpha_{j}\right), \\
\min _{n \in \Omega_{m-l_{m}}} c_{n} & =\frac{\alpha_{m-l_{m}}}{\sqrt{2^{m-l_{m}}}} \prod_{j=0}^{m-l_{m}-1}\left(1-\alpha_{j}\right) .
\end{aligned}
$$

Ясно (см. (3.17)), что для любого $m \geqslant 1$

$$
\prod_{j=0}^{m-1}\left(1+\alpha_{j}\right) \leqslant \sqrt[4]{2} 2^{l_{m} / 4} \prod_{j=0}^{m-1}\left(1-\alpha_{j}\right)
$$

и поскольку $l_{m} \geqslant 0$, то тем более

$$
\prod_{j=0}^{m-1}\left(1+\alpha_{j}\right) \leqslant \sqrt[4]{2} 2^{l_{m} / 4} \prod_{j=0}^{m-l_{m}-1}\left(1-\alpha_{j}\right) .
$$

Из (3.16) следует, что для любого $m \geqslant 0$

$$
\alpha_{m} \leqslant 2^{l_{m} / 4} \alpha_{m-l_{m}},
$$

и поэтому

$$
2^{-m / 2} \alpha_{m} \leqslant 2^{-m / 2+l_{m} / 4} \alpha_{m-l_{m}} .
$$

Из (3.20) и (3.21) для любого $m \geqslant 1$ получаем

$$
2^{-m / 2} \alpha_{m} \prod_{j=0}^{m-1}\left(1+\alpha_{j}\right) \leqslant \sqrt[4]{2} 2^{\left(-m+l_{m}\right) / 2} \alpha_{m-l_{m}} \prod_{j=0}^{m-l_{m}-1}\left(1-\alpha_{j}\right),
$$

т.е. (см. (3.18) и (3.19)) для любого $m \geqslant 1$

$$
\max _{n \in \Omega_{m}} c_{n} \leqslant \sqrt[4]{2} \min _{n \in \Omega_{m-l_{m}}} c_{n}
$$

и $c_{n}>0$ при $n \geqslant 1$.

Теорема 8 доказана. 
ДОКАЗАТЕЛЬСТВО ТЕОРЕМЫ 9 . ПолОЖим $\tau_{m}=4 \log _{2} d_{m}$. Поскольку $d_{m} \geqslant 1$ и $\lim _{m \rightarrow \infty} d_{m}=\infty$, то $\tau_{m} \geqslant 0$ и $\lim _{m \rightarrow \infty} \tau_{m}=\infty$. Поэтому в силу леммы 8 сушествует последовательность $\left\{\alpha_{j}\right\} \in S_{0}$ такая, что при $m \geqslant 1$

$$
\begin{gathered}
\alpha_{m} \leqslant \sqrt[4]{2} \alpha_{m-1}, \\
\prod_{j=0}^{m-1} \frac{1+\alpha_{j}}{1-\alpha_{j}} \leqslant 2^{\left(1+\tau_{m}\right) / 4}=\sqrt[4]{2} d_{m} .
\end{gathered}
$$

Пусть для этой последовательности $\left\{\alpha_{j}\right\}_{j=0}^{\infty}$ ряд

$$
\sum_{n=1}^{\infty} c_{n} \chi_{n}(t)
$$

представляет произведение (2.5). Тогда в силу леммы 6 ряд (2.8) есть нуль-ряд, а из леммы 3 следует, что $c_{n}>0$ при $n \geqslant 1$, а для любого $m \geqslant 2$

$$
\max _{n \in \Omega_{m}} c_{n}=\frac{\alpha_{m}}{\sqrt{2^{m}}} \prod_{j=0}^{m-1}\left(1+\alpha_{j}\right)
$$

И

$$
\min _{n \in \Omega_{m-1}} c_{n}=\frac{\alpha_{m-1}}{\sqrt{2^{m-1}}} \prod_{j=0}^{m-2}\left(1-\alpha_{j}\right)
$$

Из (3.22) при $m \geqslant 1$ имеем

$$
2^{-m / 2} \alpha_{m} \leqslant 2^{1 / 4-m / 2} \alpha_{m-1},
$$

а из (3.23) следует, что для любого $m \geqslant 2$

$$
\prod_{j=0}^{m-1}\left(1+\alpha_{j}\right) \leqslant \sqrt[4]{2} d_{m} \prod_{j=0}^{m-2}\left(1-\alpha_{j}\right)
$$

Из (3.26) и (3.27) для любого $m \geqslant 2$ следует, что

$$
2^{-m / 2} \alpha_{m} \prod_{j=0}^{m-1}\left(1+\alpha_{j}\right) \leqslant d_{m} 2^{(-m+1) / 2} \alpha_{m-1} \prod_{j=0}^{m-2}\left(1-\alpha_{j}\right),
$$

т.е. для любого $m \geqslant 2$ (см. $(3.24)$ и (3.25))

$$
\max _{n \in \Omega_{m}} c_{n} \leqslant d_{m} \min _{n \in \Omega_{m-1}} c_{n}
$$

и $c_{n}>0$ при $n \geqslant 1$. Остается показать, что (3.28) выполняется и при $m=1$, т.е.

$$
\max _{n \in \Omega_{1}} c_{n} \leqslant d_{1} \min _{n \in \Omega_{0}} c_{n}
$$


Отметим, что $\Omega_{0}=\{2\}$ и $\Omega_{1}=\{3,4\}$. В силу леммы 3

$$
\min _{n \in \Omega_{0}} c_{n}=c_{2}=\alpha_{0}
$$

и

$$
\max _{n \in \Omega_{1}} c_{n}=\frac{\alpha_{1}}{\sqrt{2}}\left(1+\alpha_{0}\right) .
$$

Ясно, что (3.29) будет выполняться, если покажем, что

$$
\frac{\alpha_{1}}{\sqrt{2}}\left(1+\alpha_{0}\right)<\alpha_{0}
$$

Действительно, из определения последовательности $\left\{\alpha_{j}\right\}$ (см. лемму 8, соотношения (2.91) и (2.104)) имеем

$$
\alpha_{m}=\frac{1}{64 m_{1}}, \text { если } 0 \leqslant m \leqslant m_{1}, \text { а } m_{1} \geqslant 1 .
$$

Отсюда, в частности, следует, что

$$
\alpha_{0}=\alpha_{1} \leqslant \frac{1}{64}
$$

Поэтому ясно, что (3.30) выполняется, и тем самьм для любого $d_{1} \geqslant 1$ справедливо соотношение (3.29).

Теорема 9 доказана.

Из доказанных теорем вытекают разные следствия. Приведем некоторые следствия теоремы 4.

Пусть $\gamma \in(-\infty,+\infty)$ - любое действительное число. Положим

$$
F_{\gamma}(u)=\left\{\begin{array}{lll}
u^{\gamma} & \text { при } & u>0 \\
0 & \text { при } & u=0
\end{array}\right.
$$

Ясно, что для любого числа $\delta \in(0,1)$ имеем: $0 \leqslant F_{\gamma}(u)<1$ при $u \in[0, \delta)$; кроме того, если $\alpha \in[0, \delta)$ и $\beta \in[0, \delta)$, то

$$
F_{\gamma}(\alpha \beta)=F_{\gamma}(\alpha) F_{\gamma}(\beta) \text { и } F_{\gamma}(0)=0,
$$

т.е. функция $F_{\gamma}(u)$ удовлетворяет требованиям теоремы 4 . Поэтому получаем справедливость следующего утверждения.

СлЕДСТВИЕ 1. Пусть $\gamma \in(-\infty,+\infty)$ - некоторое число. Тогда для любого числа $\delta \in(0,1)$ и любой последовательности $\left\{d_{m}\right\}, d_{m}>0$ u $d_{m} \uparrow \infty$, существует нуль-ряд

$$
1+\sum_{n=1}^{\infty} a_{n} \cos n \tau
$$

такой, что $0 \leqslant a_{n}<\delta$ при $n \geqslant 1$ и для кажсого $m \geqslant 1$

$$
\sum_{n=1}^{m} F_{\gamma}\left(a_{n}\right) \leqslant d_{m}
$$


СлЕДСТвИЕ 2. Пусть $\Psi(u)-$ любая функиия $c \Psi(0)=0$. Если для некоторого $\delta \in(0,1)$ функиия $\Psi(u)$ ограничена на $[0, \delta)$, то для любой последовательности $\left\{d_{m}\right\}, d_{m}>0$ и $d_{m} \uparrow \infty$, существует нуль-ряд (3.31) такой, что $0 \leqslant a_{n}<\delta$ при $n \geqslant 1$ и для каждого $m \geqslant 1$

$$
\sum_{n=1}^{m}\left|\Psi\left(a_{n}\right)\right| \leqslant d_{m}
$$

ДокАЗАТЕЛЬСТво. В силу условия для некоторых чисел $\delta \in(0,1)$ и $M>0$

$$
|\Psi(u)|<M \text { при } u \in[0, \delta) .
$$

Не ограничивая обшности, будем считать, что $M \geqslant 1$. Положим

$$
\bar{\Psi}(u)=M \operatorname{sign} u, \quad \text { где } u \in[0, \delta) .
$$

Ясно, что при $u \in[0, \delta)$

$$
|\Psi(u)| \leqslant \bar{\Psi}(u) .
$$

Кроме того, $\bar{\Psi}(0)=0$ и для $\alpha \in[0, \delta)$ и $\beta \in[0, \delta)$

$$
\bar{\Psi}(\alpha \beta)=M \operatorname{sign} \alpha \beta \leqslant M \operatorname{sign} \alpha M \operatorname{sign} \beta=\bar{\Psi}(\alpha) \bar{\Psi}(\beta),
$$

т.е. $\bar{\Psi}(u)$ удовлетворяет требованиям теоремы 4 . Поэтому в силу теоремы 4 существует нуль-ряд (3.31) такой, что $0 \leqslant a_{n}<\delta$ при $n \geqslant 1$ и для любого $m \geqslant 1$

$$
\sum_{n=1}^{m} \bar{\Psi}\left(a_{n}\right) \leqslant d_{m} .
$$

Из (3.32) и (3.33) следует справедливость следствия 2.

СлЕДСТвИЕ 3. Для любой последовательности $\left\{d_{m}\right\}, d_{m}>0 u d_{m} \uparrow \infty$, существует нуль-ряд (3.31) такой, что $0 \leqslant a_{n}<1$ при $n \geqslant 1$ и для кажсдого $m \geqslant 1$

$$
\sum_{n=1}^{m} \operatorname{sign} a_{n} \leqslant d_{m}
$$

ДокАЗАТЕЛЬСТВо. Поскольку функция $\Psi(u)=\operatorname{sign} u$ ограничена и $\Psi(0)=0$, то из следствия 2 (взяв $\delta \in(0,1))$ получаем справедливость следствия 3.

СлеДСТВИЕ 4. Пусть $\left\{q_{n}\right\}$ - любая последовательность положительных чисел, $a\left\{d_{m}\right\}$ - любая последовательность с $d_{m}>0$ u $d_{m} \uparrow \infty$. Тогда существует нуль-ряд (3.31) такой, что $a_{n} \geqslant 0$ при $n \geqslant 1$ и для каждого $m \geqslant 1$

$$
\sum_{n=1}^{m} a_{n}^{q_{n}} \leqslant d_{m} .
$$


ДоКАЗАТЕЛЬСТво. В силу следствия 3 сушествует нуль-ряд (3.31) такой, что $0 \leqslant a_{n}<1$ при $n \geqslant 1$ и для любого $m \geqslant 1$

$$
\sum_{n=1}^{m} \operatorname{sign} a_{n} \leqslant d_{m}
$$

Далее, поскольку $q_{n}>0$ и $0 \leqslant a_{n}<1$ при $n \geqslant 1$, то

$$
0 \leqslant a_{n}^{q_{n}} \leqslant \operatorname{sign} a_{n} .
$$

Из (3.35) и (3.34) вытекает справедливость следствия 4.

СлЕДСТвИЕ 5. Пусть $\Psi(u)-$ любая функция $c$

$$
\lim _{u \rightarrow 0+} \Psi(u)=\Psi(0)=0 .
$$

Тогда для любой последовательности $\left\{d_{m}\right\}, d_{m}>0 u d_{m} \uparrow \infty$, существует нуль-ряд (3.31) такой, что $a_{n} \geqslant 0$ при $n \geqslant 1$ и для каждого $m \geqslant 1$

$$
\sum_{n=1}^{m}\left|\Psi\left(a_{n}\right)\right| \leqslant d_{m} .
$$

ДокАЗАТЕЛЬСТво. В силу условия (3.36) для некоторого $\delta \in(0,1)$ выполняется соотношение:

$$
|\Psi(u)|<1, \text { если } 0 \leqslant u<\delta .
$$

Отсюда в силу следствия 2 получаем справедливость следствия 5.

ЗАмечАниЕ 2. Очевидно, что из теоремы 5 , проведя точно такие же рассуждения, как при доказательстве следствий 1-5, получим справедливость этих следствий и для нуль-рядов по системе Уолша.

Пусть $S_{m}(t)$ (как и в теоремах 6 и 7 ) обозначает частичные суммы ряда

$$
\sum_{n=1}^{\infty} a_{n} \varphi_{n}(t) .
$$

Приведем некоторые следствия теоремы 7.

СлЕДСТВИЕ 6 . Если неотрицательная, измеримая на $[0,+\infty)$ функиия $G(u)$ удовлетворяет соотношению

$$
\sup _{0 \leqslant u \leqslant A} G(u)<\infty \quad \text { npu } \quad A \geqslant 0
$$

(в частности, если $G(u)$ неотрицательна и непрерьвна на $[0,+\infty)$ ), то для любой последовательности $\left\{\varepsilon_{m}\right\}, \varepsilon_{m}>0 u \lim _{m \rightarrow \infty} \varepsilon_{m}=0$, существует нуль-ряд (3.37) такой, что при $m \geqslant 1$

$$
\int_{a}^{b}\left|S_{m}(t)\right|\left[G\left(\left|S_{m}(t)\right|\right)\right]^{\varepsilon_{m}} d t \leqslant M
$$

əде $M$ - некоторое положительное число. 
ДОКАЗАТЕЛЬСТВО. В силу условия $G(u) \geqslant 0$ при $u \geqslant 0$ и для любого $A>0$

$$
\sup _{0 \leqslant u \leqslant A} G(u)<\infty
$$

(очевидно, что этому соотношению удовлетворяет любая неотрицательная и непрерывная на $[0,+\infty)$ функция $G(u))$.

Пусть

$$
\bar{G}(0)=G(0) \text { и } \bar{G}(u)=\sup _{0 \leqslant \eta \leqslant u} G(\eta) \text { при } u>0 .
$$

Тогда ясно, что

$$
0 \leqslant \bar{G}(u)<\infty \text { при } u \geqslant 0,
$$

и если $u_{1} \leqslant u_{2}$, то $\bar{G}\left(u_{1}\right) \leqslant \bar{G}\left(u_{2}\right)$, т.е. функция $\bar{G}(u)$ удовлетворяет требованиям теоремы 7 . Поэтому в силу теоремы 7 сушествует нуль-ряд (3.37) такой, что при $m \geqslant 1$

$$
\int_{a}^{b}\left|S_{m}(t)\right|\left[\bar{G}\left(\left|S_{m}(t)\right|\right)\right]^{\varepsilon_{m}} d t \leqslant M,
$$

где $M$ - некоторое положительное число. Из определения функции $\bar{G}(u)$ следует, что $0 \leqslant G(u) \leqslant \bar{G}(u)$ для любого $u \geqslant 0$. Поэтому из (3.38) при $m \geqslant 1$ имеем

$$
\int_{a}^{b}\left|S_{m}(t)\right|\left[G\left(\left|S_{m}(t)\right|\right)\right]^{\varepsilon_{m}} d t \leqslant \int_{a}^{b}\left|S_{m}(t)\right|\left[\bar{G}\left(\left|S_{m}(t)\right|\right)\right]^{\varepsilon_{m}} d t \leqslant M .
$$

Следствие 6 доказано.

СлЕДСТВИЕ 7. Для любой последовательности $\left\{\varepsilon_{m}\right\}, \varepsilon_{m} \geqslant 0 u \lim _{m \rightarrow \infty} \varepsilon_{m}=0$, существует нуль-ряд (3.37) такой, что при $m \geqslant 1$

$$
\left\|S_{m}\right\|_{L_{1+\varepsilon_{m}}} \leqslant M
$$

әде $M$ - некоторое положительное число.

ДокАЗАТЕЛЬСтво. В теореме 7 , взяв $G(u)=u$ при $u \geqslant 0$, получаем, что сушествует нуль-ряд (3.37) такой, что при $m \geqslant 1$

$$
\int_{a}^{b}\left|S_{m}(t)\right|^{1+\varepsilon_{m}} d t \leqslant M \quad(M>0-\text { некоторое число }) .
$$

Очевидно, что, не ограничивая общности, можем считать, что $M \geqslant 1$. Поскольку $\varepsilon_{m} \geqslant 0$, то из (3.39) получаем, что при $m \geqslant 1$

$$
\left\|S_{m}\right\|_{L_{1+\varepsilon_{m}}} \leqslant M^{1 /\left(1+\varepsilon_{m}\right)} \leqslant M .
$$

Следствие 7 доказано.

ЗАМЕчАнИЕ 3. Ясно, что теоремы 6 и 7 и следствия 6 и 7 являются отрицательным решением более общего вопроса, чем приведенная во введении (см. пункт II) задача Литтлвуда. При этом этот вопрос решается как для тригонометрических рядов, так и для рядов по системам Уолша и Хаара. 


\section{Список литературы}

1. Menshoff D. E. Sur l'unicité du developpement trigonometrique // CR. 1916. V. 163. P. 433-436.

2. Шнейдер A. A. О единственности разложений по системе функций Уолша // Матем. сб. 1949. T. 24 (66). C. $279-300$.

3. Faber G. Über die Orthogonalfunktionen des Herrn Haar // Jahresb. Deutsch. Math. Verein. 1910. V. 19. P. 104-112.

4. Бари Н. К. Тригонометрические ряды. М.: Физматгиз, 1961.

5. Скворцов B. A. О скорости стремления к нулю коэффициентов нуль-рядов по системам Хаара и Уолша // Изв. АН СССР. Сер. матем. 1977. Т. 41. № 3. С. 703-716.

6. Петровская М. Б. О нуль-рядах по системе Хаара и множествах единственности // Изв. АН СССР. Сер. матем. 1964. Т. 28. С. 773-798.

7. Ульянов П. Л. Решенные и нерешенные проблемы теории тригонометрических и ортогональных рядов // УМН. 1964. Т. 19. №1. С. 3-69.

8. Weiss Mary. On a problem of Littlewood // J. London Math. Soc. 1959. V. 34. № 2. P. 217-221.

9. Ульянов П. Л. О рядах по системе Хаара с монотонньми коэффициентами // Изв. АН СССР. Сер. матем. 1964. Т. 28. С. 925-950.

10. Ульянов П. А. Об единственности рядов по системе Хаара с монотонньми коэффициентами // Вест. МГУ. Сер. 1, матем., механика. 1983. №6. С. 64-73.

11. Zygmund A. On lacunary trigonometric series // Trans. Amer. Math. Soc. 1932. V. 34. P. $435-446$.

12. Зигмунд А. Тригонометрические ряды. Т. І. М.: Мир, 1965.

13. Тетунашвили ШІ. Т. О нуљ-рядах по системе Хаара // Сообщения АН Грузии. 1991. T. 142. № 2. C. 245-247.

Грузинский технический университет

Поступила в редакцию

28.04 .1995 